\title{
Collisional and dynamical evolution of the main belt and NEA population
}

\author{
G. C. de Elía and A. Brunini \\ Facultad de Ciencias Astronómicas y Geofísicas, Universidad Nacional de La Plata, Paseo del Bosque S/N (1900), La Plata, Argentina \\ IALP-CONICET \\ e-mail: [gdeelia, abrunini]@fcaglp.unlp.edu.ar
}

Received 14 July 2006 / Accepted 15 January 2007

\begin{abstract}
Aims. In this paper, we analyze the collisional evolution of the Main Belt and NEA population taking into account the major dynamical features present in both populations.

Methods. To do this, we divide the asteroid belt into three semimajor axis zones, whose boundaries are given by the $v_{6}$ secular resonance, and the 3:1, 5:2 and 2:1 mean motion resonances with Jupiter, treating them as strong sources of dynamical removal. We also consider the action of the Yarkovsky effect and diffusive resonances as mechanisms of mass depletion. This treatment allows us to calculate the direct collisional injection into the powerful resonances, to study the collisional exchange of mass between the different regions of the Main Belt and to analyze the provenance of the NEA objects.

Results. Our model is in agreement with the major observational constraints associated with the Main Belt and NEA populations, such as their size distributions, the collisional history of Vesta, the number of large asteroid families and the cosmic-ray exposure (CRE) ages of meteorites. We find that none of the dynamical and collisional mechanisms included in our treatment are able to mix material between the three studied main belt regions, since more than $99 \%$ of the final mass of every ring of our model of the Main Belt is represented by primordial material. In addition, our results supports that the Yarkovsky effect is the most important process that removes material from the asteroid Main Belt, rather than collisional injection into the major resonances. With regards to the provenance of the NEAs, our work shows that $\sim 94 \%$ of the NEA population comes from the region inside the 5:2 mean motion resonance.
\end{abstract}

Key words. minor planets, asteroids - methods: $N$-body simulations - methods: numerical - solar system: formation

\section{Introduction}

The Main Belt of asteroids is a vast region located between Mars and Jupiter, roughly from 2 to 3.4 AU from the Sun. The near-Earth asteroids (NEAs) represent another important population. NEAs have perihelion distances $q \leq 1.3 \mathrm{AU}$ and aphelion distances $Q \geq 0.983$ AU (Rabinowitz et al. 1994). They are customary divided into three subcategories including the Atens $(a<1 \mathrm{AU}, Q \geq 0.983 \mathrm{AU})$ and Apollos $(a \geq 1 \mathrm{AU}$, $q \leq 1.0167 \mathrm{AU})$, which are on Earth-crossing orbits, and the Amors (1.0167 AU $<q \leq 1.3 \mathrm{AU})$, which are on nearly-Earthcrossing orbits. Figure 1 shows the distribution of Main Belt asteroids and NEAs with respect to semimajor axis, eccentricity and inclination.

The Main Belt asteroids and NEAs do not represent independent populations; on the contrary, they are intimately connected by evolutionary processes and dynamical transport mechanisms associated to orbital resonances. While the existence of resonances in the Main Belt has been known since many years, most works aimed at studying the collisional evolution of the small bodies in such region has not accounted for them. Since the work of Williams (1969) and further studies developed by Wetherill (1979) and Wisdom (1983, 1985a, 1985b), it is widely accepted that the resonant regions present in the asteroid Main Belt are effective escape routes from there. In fact, detailed numerical simulations performed by Gladman et al. (1997) have shown that those objects falling into some resonance inside $2.5 \mathrm{AU}$ could become NEAs and then meteorites in only a few million of years, being the most common end state of these objects an impact onto the Sun. They have also shown that those bodies reaching one of the resonant regions outside 2.5 AU become Jupiter-crossers and are subsequently removed from the Solar System by close encounters with Jupiter. The intense collisional activity present in the asteroid Main Belt continuously breaks up large asteroids, injecting a large quantity of material into the resonant regions, a mechanism that represents a source of mass depletion in the Main Belt.

In the early 2000s, Penco et al. (2002) included the so-called Yarkovsky effect into numerical models of the asteroid collisional evolution. The Yarkovsky effect, a radiation force, modifies the orbital parameters of asteroids giving rise to a mechanism that can deliver them into resonances and thus remove them from the Main Belt. Besides, this effect is size dependent and owing to that, its action coupled with the presence of resonant regions not only can be another important source of steady mass depletion in the Main Belt, but can also affect their size distribution. There are strong evidences associated with the size distribution of the NEA population which might suggest that Yarkovsky effect is the most important process that drives asteroids into resonances and primarily into the NEA source resonances, rather than direct collisional injection (Morbidelli et al. 2002; Morbidelli \& Vokrouhlický 2003).

The arguments presented so far allow us to infer that the size distribution of NEAs is fundamentally determined by the Main Belt population from which they come and the collisional and 

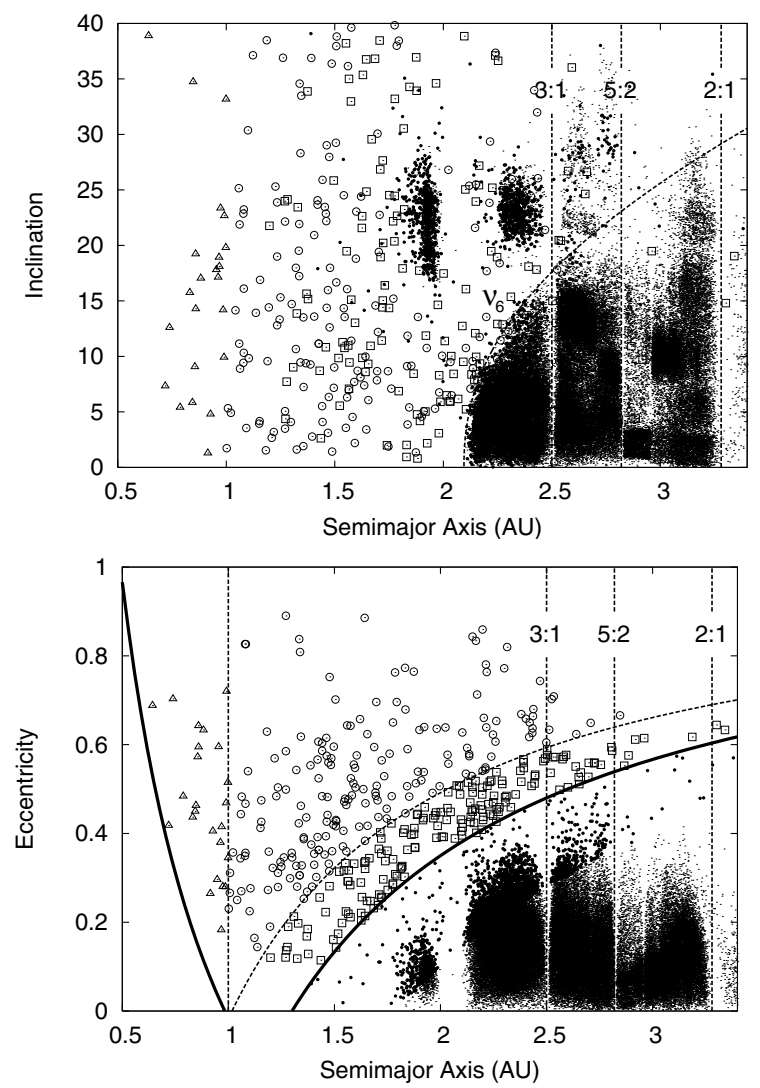

Fig. 1. The distribution of Main Belt asteroids, Mars-crossers and NEAs with respect to semimajor axis, eccentricity and inclination. The Main Belt asteroids are plotted as small dots while the Mars-crossers are represented as larger black points. The solid curves delimit the NEA region. The Atens, Apollos and Amors are shown as triangles, circles and squares, respectively. In fact, while the dashed vertical line determines the boundary between the Aten and Apollo regions, the dashed curve represents the separation between the Apollo and Amor populations. The 3:1, 5:2 and 2:1 mean motion resonances with Jupiter are labeled in both figures. On the other hand, the $v_{6}$ secular resonance is just shown on the top panel since its position depends on the orbital inclination and only weakly on the eccentricity. (Data obtained from http://ssd.jpl. nasa.gov/dat/ELEMENTS. NUMBR.)

dynamical mechanisms which are responsible for their transport. Thus, a complete model of the asteroid Main Belt and NEAs must follow the simultaneous evolution of both populations. Besides, a suitable code must be able to include collisional and dynamical processes, since without dynamical mechanisms acting, the NEA population would never be generated and, without collisional evolution, there would be no fresh collisional fragments and the bodies removed from the Main Belt could not be continuously replenished.

Recently, Bottke et al. (2005a), O’Brien \& Greenberg (2005) and Bottke et al. (2005b) developed works aimed at analyzing the evolution of the Main Belt and NEA populations. Bottke et al. (2005a) developed a collisional evolution model aimed at studying the Main Belt comminution from the end of accretion among $D<1000 \mathrm{~km}$ bodies to the present day. These authors find that the Main Belt size distribution is predominately a fossil produced in the first years of collisional evolution, when the Main Belt population was once far more massive than the current population. The work presented by Bottke et al. (2005a) has allowed to analyze some questions related to the shape of the initial Main Belt size distribution, stability of Main Belt and
NEA populations, the collisional history of Vesta, asteroid disruption frequency, asteroid spin rates and the estimated size of the primordial Main Belt. On the other hand, the study developed by O'Brien \& Greenberg (2005) models the evolution of the Main Belt asteroids, the near-Earth asteroids (NEAs) and the trans-Neptunian objects (TNOs). In particular, these authors perform a self-consistent numerical code for modeling the simultaneous evolution of the Main Belt and NEA populations, considering collisional processes and dynamical mechanisms such as the Yarkovsky effect and orbital resonances. This numerical algorithm is able to satisfy the major observational constraints associated with these small-body populations, such as their size distributions, the collisional history of Vesta, the number of large asteroid families and the cosmic-ray exposure (CRE) ages of meteorites. Later, Bottke et al. (2005b) performed a study aimed at linking the collisional history of the asteroid Main Belt to its dynamical excitation and depletion. This work combines dynamical results from Petit et al. (2001) with the collisional evolution code created by Bottke et al. (2005a). The results are consistent with the Main Belt's size-frequency distribution, the number of currently observable asteroid families produced by collisional disruption events involving parent bodies larger than $100 \mathrm{~km}$, the collisional history of Vesta and the lunar and terrestrial impactor flux over the last 3 Gyr. Moreover, this model allows also to study the NEA population, which is used to explore some questions about the small craters formed on Mercury, the Moon and Mars.

Here, we present a new multi-population code for collisional evolution that takes into account the main dynamical features present in the asteroid Main Belt and NEA region. Among the works of Bottke et al. (2005a), O'Brien \& Greenberg (2005) and Bottke et al. (2005b), the second one is the most similar to that shown in this paper, though there are some relevant differences in the populations of the model, collisional input parameters and in the treatment of the dynamical evolution. In fact, the most notable difference between those papers and our work is that our model proposes to divide the asteroid belt into three semimajor axis zones whose boundaries are given by the $v_{6}, 3: 1,5: 2$ and 2:1 powerful resonances, which has allowed us to develop a more rigorous study of the Main Belt and NEA populations. We believe our model improves those presented by Bottke et al. (2005a), O'Brien \& Greenberg (2005) and Bottke et al. (2005b), allowing us to analyze some questions related with the mixing of material in the asteroid belt, the provenance of the NEA objects and the collisional injection into the powerful resonances.

In Sect. 2 the collisional model is described, while the most important dynamical mechanisms taken into account in our algorithm are presented in Sect. 3. In Sect. 4 we describe the full numerical model, while Sect. 5 shows the most important results derived from the collisional and dynamical evolution of the asteroid Main Belt and NEA population. Conclusions are given in the last section.

\section{Collisional mechanisms}

In this section, we present the main features of our algorithm aimed at describing the outcome of a collision between two bodies.

\subsection{Collisional parameters - definitions}

As it is usual, a catastrophic collision is defined as the one where the largest piece resulting from it contains $50 \%$ or less of the 
initial target mass, whereas the rest of the collisions are considered cratering events.

The impact velocity $V$ and the shattering impact specific energy $Q_{\mathrm{S}}$ are two fundamental quantities determining, for a given body, if the collision must be studied in the catastrophic regime or in the cratering regime. $Q_{\mathrm{S}}$ is the amount of energy per unit target mass needed to catastrophically fragment a body, such that the largest resulting fragment has half the mass of the original target, regardless of reaccumulation of fragments. While early works of Dohnanyi (1969), Williams \& Wetherill (1994) and Tanaka et al. (1996) assumed that all asteroids had the same impact strength per unit mass (namely, $Q_{\mathrm{S}}$ would be a constant), since more recent numerical models as well as laboratory studies it is now accepted that $Q_{\mathrm{S}}$ is size-dependent. Farinella et al. (1982), Housen \& Holsapple (1990), Ryan (1992), Holsapple (1993), Housen \& Holsapple (1999) and Benz \& Asphaug (1999) have shown that for small bodies, with diameters $\lesssim 1 \mathrm{~km}$, the material properties control the impact strength in such a way that it decreases with increasing size. On another hand, Davis et al. (1985), Housen \& Holsapple (1990), Love \& Ahrens (1996), Melosh \& Ryan (1997), and Benz \& Asphaug (1999) showed that for large asteroids, with diameters $\gtrsim 1 \mathrm{~km}$, gravity dominates the impact strength which increases with increasing size. Some authors (Durda et al. 1998) have used $Q_{\mathrm{D}}$ (the amount of energy per unit mass needed to fragment a body and disperse half of its mass) rather than $Q_{\mathrm{S}}$, as primary input parameter in their collisional evolution models. For small bodies, the gravitational binding energy is negligible and owing to that $Q_{\mathrm{S}}$ and $Q_{\mathrm{D}}$ have the same value. For larger bodies, $Q_{\mathrm{D}}$ must be larger than $Q_{\mathrm{S}}$, since gravity is important and can therefore impede the dispersal of fragments. In Sect. 4.3, we will discuss some aspects of $Q_{\mathrm{S}}$ and $Q_{\mathrm{D}}$, specifying the most convenient input parameters for our collisional evolution model.

On the other hand, the relative kinetic energy in a collision between two bodies of masses $M_{1}$ and $M_{2}$ is given by

$E_{\text {rel }}=\frac{1}{2} \frac{M_{1} M_{2}}{M_{1}+M_{2}} V_{\text {rel }}^{2}$

where $V_{\text {rel }}$ is the relative impact velocity.

According to these definitions and assuming that the energy is equi-partitioned between the two colliding bodies (Hartmann 1988 ), for body $i$ fragmentation occurs if $E_{\mathrm{rel}}>2 Q_{\mathrm{S}, i} M_{i}$ (Greenberg et al. 1978; Petit \& Farinella 1993), while below this threshold, cratering happens. Thus, if two objects collide, the last relation allows us to determine if both of them will be catastrophically fragmented, if one will be cratered and the other will be catastrophically fragmented or if both will be cratered after the collision.

In the next subsections, we will describe our treatment of a collision in the catastrophic regime as well as in the cratering regime. Besides, for any of the three mentioned outcomes, we also study the escape and reaccumulation processes of the resulting fragments, carrying out a previous determination of the escape velocity.

\subsection{Catastrophic fragmentation}

In order to model the distribution of the fragments resulting from a catastrophic fragmentation event, we develop a model based on Petit \& Farinella's (1993) algorithm. These authors use a single-slope power law to describe the fragment distribution from a catastrophic event. O'Brien \& Greenberg's (2005) collisional algorithm is also based on Petit \& Farinella's (1993) method but introduces a two-slope power law to model the distribution of fragments resulting from a catastrophic fragmentation, which is a more realistic description according to laboratory experiments and hydrocode models. But, O’Brien \& Greenberg (2005) show that using a two-slope power law rather than a less realistic single-slope power law obtains a worse fit in the simulations, which probably indicates a limitation of the collisional model rather than suggesting that asteroids are catastrophically fragmented following a single-slope power law. From these results, we decide to use a single-slope power law to describe the distribution of fragments resulting from a catastrophic event.

If a body of mass $M_{i}$ is catastrophically fragmented, the mass of the largest resulting fragment will be given by $M_{\max , i}=M_{i} f_{l, i}$, where $f_{l, i}$ is

$f_{l, i}=\frac{1}{2}\left(\frac{Q_{\mathrm{S}, i} M_{i}}{E_{\mathrm{rel}} / 2}\right)^{1.24}$,

according to the experimental results obtained by Fujiwara et al. (1977).

We define $N_{i}(\geq m)$ as the number of fragments of body $i$ with a mass larger than $m . N_{i}(\geq m)$ has a discontinuity at $m=M_{\max , i}$ since there is just one fragment of mass $M_{\max , i}$ resulting from the catastrophic fragmentation of body $i$. So, if $\Theta(x)$ is the Heaviside step function (namely, $\Theta(x)=0$ for $x<0$ and $\Theta(x)=1$ for $x \geq$ $0), N_{i}(\geq m)$ can be written as

$N_{i}(\geq m)=B_{i} m^{-b_{i}} \Theta\left(M_{\max , i}-m\right)$,

where $b_{i}$ is the characteristic exponent. Besides, as $N_{i}\left(\geq M_{\max , i}\right)=1$, so from the last equation, we find $B_{i}=\left(M_{\mathrm{max}, i}\right)^{b_{i}}$. In order to calculate the characteristic exponent $b_{i}$, we derive the cumulative mass distribution $M_{i}(\leq m)$ which represents the total mass of fragments of body $i$ with a mass smaller than $m$. In fact, $M_{i}(\leq m)$ can be calculated as

$M_{i}(\leq m)=\int_{0}^{m} m n_{i}(m) \mathrm{d} m$,

where $n_{i}(m) \mathrm{d} m=-\mathrm{d} N_{i}(\geq m)$ defines the differential fragment size distribution. According to Eq. (3), $n_{i}(m) \mathrm{d} m$ will be given by

$$
\begin{aligned}
n_{i}(m) \mathrm{d} m= & \left\{b_{i} B_{i} m^{-b_{i}-1} \Theta\left(M_{\max , i}-m\right)\right. \\
& \left.+B_{i} m^{-b_{i}} \delta\left(m-M_{\max , i}\right)\right\} \mathrm{d} m,
\end{aligned}
$$

where $\delta(x)=\mathrm{d} \Theta(x) / \mathrm{d} x$ is the Dirac delta function. Then, inserting it in Eq. (4) and using that $B_{i}=\left(M_{\max , i}\right)^{b_{i}}$, so $M_{i}(\leq m)$ will be written as

$$
\begin{aligned}
M_{i}(\leq m)= & \frac{b_{i} M_{\max , i}^{b_{i}}}{1-b_{i}} m^{1-b_{i}}\left\{1-\Theta\left(m-M_{\max , i}\right)\right\} \\
& +\frac{M_{\max , i}}{1-b_{i}} \Theta\left(m-M_{\max , i}\right) .
\end{aligned}
$$

From this equation, it is possible to derive a relation between $f_{l, i}$, given by Eq. (2), and the characteristic exponent $b_{i}$. Actually, the mass conservation implies $M_{i}\left(\leq M_{\max , i}\right)=M_{i}$; then, from Eq. (6), we derive the condition

$M_{i}=\frac{M_{\max , i}}{1-b_{i}}$

and, since $M_{\max , i}=M_{i} f_{l, i}$, so

$b_{i}=1-f_{l, i}$.

Thus, if $f_{l, i}$ is calculated by Eq. (2), $b_{i}$ can be derived from the last equation. With this, every parameter present in Eq. (3) is determined and so, such law can be used in order to calculate the distribution of the fragments resulting from a catastrophic event. 


\subsection{Cratering impacts}

Below the catastrophic fragmentation threshold $\left(E_{\mathrm{rel}}<2 Q_{\mathrm{S}, i} M_{i}\right)$, a crater is formed. Again, we use Petit \& Farinella's (1993) algorithm in order to calculate the distribution of fragments resulting from a cratering event. Imposing continuity for $M_{\mathrm{crat}, i}=M_{i} / 100$, the mass $M_{\text {crat }, i}$ excavated from the crater can be calculated from the following relations

$M_{\mathrm{crat}, i}= \begin{cases}\alpha E_{\mathrm{rel}} & \text { if } E_{\mathrm{rel}} \leq \beta, \\ \frac{9 \alpha}{200 Q_{\mathrm{S}, i} \alpha-1} E_{\mathrm{rel}}+\frac{M_{i}}{10} \frac{1-20 Q_{\mathrm{S}, i} \alpha}{1-200 Q_{\mathrm{S}, i} \alpha} & \text { if } E_{\mathrm{rel}}>\beta,\end{cases}$

where $\beta=M_{i} / 100 \alpha$. The parameter $\alpha$, known as crater excavation coefficient, depends on the material properties and ranges from about $4 \times 10^{-4}$ to $10^{-5} \mathrm{~s}^{2} \mathrm{~m}^{-2}$ for soft and hard materials respectively (Stoeffler et al. 1975; Dobrovolskis \& Burns 1984). As Eq. (9) indicates, the model proposed by Petit \& Farinella (1993) assumes a linear dependence of $M_{\text {crat }, i}$ on $E_{\text {rel }}$ in such a way that for craters smaller than $1 \%$ of the target mass, $M_{\mathrm{crat}, i}$ is proportional to $E_{\text {rel }}$ while for larger craters, the coefficients of the linear relation are chosen such that the largest possible crater has a mass of $1 / 10$ that of the target, which is in agreement with the studies developed by Nolan et al. (1996).

For cratering impacts, the surviving cratered body has a mass $M_{i}-M_{\text {crat }, i}$. As in the case of a catastrophic fragmentation event, we also assume a single-slope power law for the fragment size distribution resulting from a cratering impact. It is important to take into account that the derived expressions to treat a catastrophic impact can be used in order to study a cratering event, replacing the target mass $M_{i}$ by $M_{\text {crat }, i}$. Thus, the mass of the largest fragment ejected from the crater will be $f_{l, i} M_{\mathrm{crat}, i}$, where $f_{l, i}=0.2$ since according to Melosh (1989), $b_{i}=0.8$ for any cratering event.

\subsection{Escape and reaccumulation of fragments}

After calculating the distribution of fragments associated with every one of bodies that participate in a collision, it is necessary to determine the final fate of the fragments ejected from each one of them. If the fragment relative velocity is larger than the escape velocity $V_{\text {esc }}$ from the two colliding bodies, it will escape, while those slower than $V_{\text {esc }}$ will be reaccumulated on the largest remnant. The following points must be considered:

- To adopt a Fragment Velocity Distribution.

- To determine the Escape Velocity of the Fragments.

According to Campo Bagatin et al. (1994b), it is possible to adopt two different models in order to study the escape of fragments and reaccumulation:

1. A "Cumulative Model", in which there is no relation between mass and velocity of fragments. This model just assumes that the fraction of the fragment mass ejected from body $i$ with speeds larger than a value $V$ is given by

$f(\geq V)=\frac{M_{i}(\geq V)}{M_{i}}=\left(\frac{V}{V_{\min }}\right)^{-k}$,

where $V_{\min }$ is a lower cutoff for the velocity of fragments. Gault et al. (1963) observed such a relationship, with a value of $k$ of about $9 / 4$.

2. As Petit \& Farinella (1993) proposed, a "Mass-Velocity Model". According to experimental results (Nakamura \& Fujiwara 1991; Nakamura et al. 1992; Giblin et al. 1994;
Giblin 1998), this model assumes that there is a correlation between the ejection velocity and the mass of the fragments. One can express the mass-velocity distribution as

$V=C_{i} m^{-r_{i}}$,

where $C_{i}$ is a constant coefficient and $r_{i}$ is a given exponent.

It is possible to find a relationship between the cumulative velocity distribution exponent $k$ and the exponent $r_{i}$ in the massvelocity model. For this, from Eq. (11), we write $m(V)$ as

$m(V)=\left(\frac{V}{C_{i}}\right)^{-1 / r_{i}}$

Inserting it in the cumulative mass distribution given by Eq. (6) and considering masses smaller than $M_{\max , i}$, we have that

$$
\begin{aligned}
M_{i}(\geq V) & =\frac{b_{i} M_{\max , i}^{b_{i}}}{1-b_{i}} m(V)^{1-b_{i}} \\
& =\frac{b_{i} M_{\max , i}^{b_{i}}}{1-b_{i}}\left(\frac{V}{C_{i}}\right)^{-\left(1-b_{i}\right) / r_{i}} .
\end{aligned}
$$

Equations (10) and (13) allow us to see that

$r_{i}=\frac{1-b_{i}}{k}$

where $b_{i}$ is obtained from Eq. (8) for a catastrophic collision or it is equal to 0.8 for a cratering event.

Here, we follow the method of Petit \& Farinella (1993) to calculate the velocity distribution of fragments. The massvelocity distribution can be written as

$$
\begin{aligned}
& V=C_{i} m^{-r_{i}} \quad \text { for } \quad \bar{M}_{i} \leq m \leq M_{\max , i}, \\
& V=V_{\max } \quad \text { for } \quad m<\bar{M}_{i},
\end{aligned}
$$

where, imposing continuity, $\bar{M}_{i}=\left(V_{\max } / C_{i}\right)^{-1 / r_{i}} . V_{\max }$ is assumed to be the maximum value for the velocity of the fragments. The inclusion of this high velocity cutoff is motivated by a physical reason: a fragment can not be ejected with a velocity larger than the sound speed in the material, which is assumed to be of $3000 \mathrm{~m} \mathrm{~s}^{-1}$ (O'Brien \& Greenberg 2005). While this value would seem to be too large (Vokrouhlický et al. 2006), in Sect. 5.4, we will discuss the dependence of our simulations on this input parameter. On the other hand, the constant coefficient $C_{i}$ can be calculated from an energy conservation equation. Assuming that the relative kinetic energy $E_{\text {rel }}$ of the collision is partitioned equally between the target and the projectile, so body $i$ will receive an energy $E_{i}=E_{\mathrm{rel}} / 2$ at impact. From this, we define $E_{\mathrm{fr}, i}=f_{\mathrm{ke}} E_{i}$ as the kinetic energy of the fragments resulting from such body. $f_{\mathrm{ke}}$ is an inelasticity parameter determining which fraction of the energy received by a body is partitioned into kinetic energy of the fragments. In Sect. 4.3, we will discuss some aspects of this parameter. On the other hand, while $E_{\mathrm{fr}, i}=f_{\mathrm{ke}} E_{i}$, it can be also written following the mass-velocity model proposed. In fact,

$$
\begin{aligned}
E_{\mathrm{fr}, i}= & \lim _{\epsilon \rightarrow 0} \int_{\bar{M}_{i}}^{M_{\max , i}-\epsilon} \frac{V^{2}}{2} m n_{i}(m) \mathrm{d} m+\frac{V_{\max }^{2}}{2} M\left(\leq \bar{M}_{i}\right) \\
& +\lambda_{i} \frac{V_{l f, i}^{2}}{2} M_{\max , i}
\end{aligned}
$$

where $n_{i}(m) \mathrm{d} m=-\mathrm{d} N_{i}(\geq m)$ is given by Eq. (5), and the last term is the kinetic energy of the largest fragment resulting from body $i$ 
in a collision. The experimental studies performed by Fujiwara \& Tsukamoto (1980) and Nakamura \& Fujiwara (1991), indicate that the largest fragment resulting from a catastrophic fragmentation event has a negligible kinetic energy in the reference frame of the center of mass. On the other hand, in a cratering event, the largest fragment of mass $M_{\mathrm{max}, i}=f_{l, i} M_{\mathrm{crat}, i}$ (with $f_{l, i}=0.2$ ) has a velocity $V_{l f, i}$ given by

$V_{l f, i}=C_{i} M_{\max , i}^{-r_{i}}$.

So, in order to take into account this difference, we insert the corresponding term in the energy conservation equation multiplied by a factor $\lambda_{i}$, where $\lambda_{i}$ will be 0 for a catastrophic event and 1 for a cratering event.

Equation (16) is an integral of $m$. Since the integrand is written in terms of $\delta\left(m-M_{\max , i}\right)$ by Eq. (5), if one wants to solve this integral over the range $\left(\bar{M}_{i}, M_{\max , i}\right)$, it is necessary to introduce epsilon and take the limit for epsilon to zero. Once $V$ is written in terms of $m$ (Eq. (15)), such integral can be evaluated. Thus

$$
\begin{aligned}
E_{\mathrm{fr}, i}= & \lambda_{i} C_{i}^{2} \frac{M_{\mathrm{max}, i}^{1-2 r_{i}}}{2}+\frac{V_{\max }^{2}}{2} \frac{b_{i}}{1-b_{i}} M_{\max , i}^{b_{i}} \bar{M}_{i}{ }^{1-b_{i}} \\
& +\frac{C_{i}^{2}}{2} \frac{b_{i} M_{\max , i}^{b_{i}}}{1-b_{i}-2 r_{i}}\left[M_{\max , i}^{1-b_{i}-2 r_{i}}-\bar{M}_{i}{ }^{1-b_{i}-2 r_{i}}\right] \\
= & C_{i}^{k_{i}} b_{i} \frac{M_{\max , i}^{b_{i}} V_{\max }^{2-k_{i}}}{2}\left[\frac{1}{1-b_{i}}-\frac{1}{1-b_{i}-2 r_{i}}\right] \\
& +C_{i}^{2} \frac{M_{\max , i}^{1-2 r_{i}}}{2}\left[\lambda_{i}+\frac{b_{i}}{1-b_{i}-2 r_{i}}\right] .
\end{aligned}
$$

From this, the constant coefficient $C_{i}$ is given by the solution of the equation

$a C_{i}^{k_{i}}+b-C_{i}^{2}=0$

where $a$ and $b$ are given by

$$
\begin{aligned}
& a=M_{\max , i}^{2 r_{i}+b_{i}-1} V_{\max }^{2-k_{i}}\left[\frac{2 b_{i} r_{i}}{\left[\left(1-2 r_{i}-b_{i}\right) \lambda_{i}+b_{i}\right]\left(1-b_{i}\right)}\right] \\
& b=2 M_{\max , i}^{2 r_{i}-1}\left[\frac{1-b_{i}-2 r_{i}}{\left(1-2 r_{i}-b_{i}\right) \lambda_{i}+b_{i}}\right] E_{\mathrm{fr}, i},
\end{aligned}
$$

and $E_{\mathrm{fr}, i}$ is assumed to be $f_{\mathrm{ke}} E_{i}$.

Once the fragment velocity distribution has been found for each of the bodies that participate in a collision, it is necessary to calculate the effective escape velocity $V_{\text {esc }}$ from the gravitational field of the two colliding bodies. For this, we use the method developed by Petit \& Farinella (1993) with the corrections made by O'Brien \& Greenberg (2005). Thus, we calculate the escape velocity $V_{\text {esc }}$ using the energy balance equation, which can be written as

$\frac{1}{2} M^{*} V_{\mathrm{esc}}^{2}+W_{\mathrm{tot}}=W_{\mathrm{fr}, 1}+W_{\mathrm{fr}, 2}+W_{\mathrm{h}}$,

where $M^{*}=M_{1}-M_{\max , 1}+M_{2}-M_{\max , 2}$ if both bodies are catastrophically fragmented, $M^{*}=M_{\text {crat }, 1}+M_{2}-M_{\max , 2}$ if body 1 is cratered and body 2 is catastrophically fragmented and $M^{*}=$ $M_{\text {crat, } 1}+M_{\text {crat, } 2}$ if both bodies are cratered. The term $W_{\text {tot }}$ is the total gravitational potential energy of the two colliding bodies just before fragmentation event, which is given by

$W_{\mathrm{tot}}=-\frac{3 G M_{1}^{5 / 3}}{5 Q}-\frac{3 G M_{2}^{5 / 3}}{5 Q}-\frac{G M_{1} M_{2}}{Q M_{1}^{1 / 3}+Q M_{2}^{1 / 3}}$, where the parameter $Q$ is

$Q=\left(\frac{4 \pi \rho}{3}\right)^{-1 / 3}$

and $\rho$ is the density of the objects. On the other hand, the terms $W_{\mathrm{fr}, i}$ represent the gravitational potential energy of the fragments of body $i$ resulting from the collision. If body $i$ is catastrophically fragmented, $W_{\mathrm{fr}, i}$ will be given by

$$
\begin{aligned}
W_{\mathrm{fr}, i} & =-\frac{3}{5} \frac{G}{Q} \int_{m=0}^{m=\infty} m^{5 / 3} n_{i}(m) \mathrm{d} m \\
& =-\frac{3 G}{Q} \frac{M_{\mathrm{max}, i}^{5 / 3}}{5-3 b_{i}},
\end{aligned}
$$

while if body $i$ is cratered, $W_{\mathrm{fr}, i}$ will adopt the following expression

$$
\begin{aligned}
W_{\mathrm{fr}, i} & =-\frac{3}{5} \frac{G}{Q} \int_{m=0}^{m=\infty} m^{5 / 3} n_{i}(m) \mathrm{d} m-\frac{3 G\left(M_{i}-M_{\mathrm{crat}, i}\right)^{5 / 3}}{5 Q} \\
& =-\frac{G}{Q} M_{\max , i}^{5 / 3} \frac{3}{5-3 b_{i}}-\frac{3 G\left(M_{i}-M_{\mathrm{crat}, i}\right)^{5 / 3}}{5 Q} .
\end{aligned}
$$

The term $W_{\mathrm{h}}$ is an estimate of the gravitational potential energy of the fragments when these are separated by a distance of the order of the Hill's radius of the total colliding mass in the gravitational field of the central mass $M_{\mathrm{o}}$ and orbital distance $R_{\mathrm{o}}$. If both bodies are catastrophically fragmented, $W_{\mathrm{h}}$ is given by

$W_{\mathrm{h}}=-\frac{3 G\left(M_{1}+M_{2}\right)^{5 / 3}}{5} \frac{\left(3 M_{\mathrm{o}}\right)^{1 / 3}}{R_{\mathrm{o}}}$,

where $M_{\mathrm{O}}$ is the mass of the Sun and $R_{\mathrm{O}}$ is the orbital radius where the collision occurs. On the other hand, according to O'Brien \& Greenberg (2005), if body 1 is cratered and body 2 is catastrophically fragmented, the term $W_{\mathrm{h}}$ must be written as

$W_{\mathrm{h}}=-\frac{3 G\left(M_{2}+M_{\mathrm{crat}, 1}\right)\left(M_{1}-M_{\mathrm{crat}, 1}\right)^{2 / 3}}{2} \frac{\left(3 M_{\mathrm{o}}\right)^{1 / 3}}{R_{\mathrm{o}}}$,

while if both bodies are cratered, the term $W_{\mathrm{h}}$ has the form

$$
\begin{aligned}
W_{\mathrm{h}}= & -\frac{3 G\left(M_{1}+M_{2}-M_{\text {crat }, 1}-M_{\text {crat }, 2}\right)^{2 / 3}}{2} \\
& \times\left(M_{\text {crat }, 1}+M_{\text {crat }, 2}\right) \frac{\left(3 M_{\mathrm{o}}\right)^{1 / 3}}{R_{\mathrm{o}}} .
\end{aligned}
$$

Once the different $W$ terms are calculated, it is possible to find the escape velocity $V_{\text {esc }}$ from the corresponding energy balance equation. From this, in Sect. 4.5 we describe the treatment proposed in our algorithm in order to study the escape and reaccumulation processes of the ejected fragments.

\section{Dynamical mechanisms}

The population of Main Belt asteroids is determined fundamentally by collisional processes. But, as we have already said, collisions are not the only process that can play an important role in the quantitative determination of the Main Belt size distribution. In fact, there are several dynamical mechanisms which can have a relevant influence on the evolution of these small bodies. The orbital resonances between asteroids and the planets as well as the Yarkovsky effect play a dominant role in removing material from the Main Belt. Besides, these dynamical mechanisms 
Table 1. Summary of the dynamical properties of the bodies injected into the $v_{6}, 3: 1$ and 5:2 powerful resonances studied by different authors. The median lifetimes (Half-Life) of bodies initially in each of those resonances and the median times required to cross the orbit of the Earth ( $T_{\text {cr }}$ Earth) have been derived by Gladman et al. (1997). Bottke et al. (2002) determined the median times spent in the NEA region $\left(T_{\text {NEA }}\right)$ for bodies coming from the different resonant regions. On the other hand, the typical end states were analyzed by Gladman et al. (1997) who followed the orbital histories of more than one hundred particles injected into each resonance for up to $\sim 100$ Myr. Finally, the mean collision probabilities with Earth $\left(P_{\text {col }}\right.$ Earth), integrated over the lifetime in the Earth-crossing region, were derived by Morbidelli \& Gladman (1998).

\begin{tabular}{lccc}
\hline \hline & $v_{6}$ & $3: 1$ & $5: 2$ \\
\hline \multicolumn{4}{c}{ Time scales (Myr) } \\
Half-Life & 2.3 & $\sim 2.4$ & $\sim 0.6$ \\
$T_{\text {cr }}$ Earth & 0.5 & $\sim 1.2$ & $\sim 0.4$ \\
$T_{\text {NEA }}$ & 6.54 & 2.16 & 0.4 \\
\hline \multicolumn{5}{c}{ End states (\%) } \\
Impact sun & 79.1 & $\sim 71.5$ & $\sim 7.1$ \\
Hyperbolic orbit & 11.8 & $\sim 27.4$ & $\sim 90.9$ \\
Survivors & 1.8 & 0.7 & 0 \\
\hline \multicolumn{5}{c}{ Collision probability } \\
$P_{\text {col }}$ Earth & $\sim 10^{-2}$ & $2 \times 10^{-3}$ & $2.5 \times 10^{-4}$ \\
\hline \multicolumn{5}{c}{}
\end{tabular}

lead to a connection between the Main Belt and NEA population. These arguments lead us to think that any model trying to analyze the evolution of the small bodies in the Inner Solar System must include such dynamical mechanisms. The purpose of this section is to give a brief description about the most important properties of the orbital resonances in the Main Belt and the Yarkovsky effect.

\subsection{Orbital resonances}

It is now widely accepted that the orbital resonances in the asteroid Main Belt provide effective "escape routes" from there. Morbidelli et al. (2002) suggest to distinguish between "powerful resonances" and "diffusive resonances".

The powerful resonances are characterized by the existence of associated well-defined gaps in the Main Belt asteroid distribution. The $v_{6}$ secular resonance which determines the inner edge of the asteroid belt, and the mean motion resonances with Jupiter 3:1, 5:2 and 2:1 at 2.5, 2.8 and 3.27 AU from the Sun respectively, represent the most important resonances of this class (see Fig. 1). Gladman et al. (1997) studied a large number of test bodies in these resonant regions and found that the $v_{6} \mathrm{sec}-$ ular resonance and 3:1 mean motion resonance with Jupiter are important sources of NEAs, while the rest of the major resonances are not very effective in producing NEAs, although they can produce changes of the orbital elements of objects entering into them, delivering such objects to cross Jupiter's orbit. In fact, the results obtained by Gladman et al. (1997) indicate that while the majority of the powerful resonances are important sources of mass depletion in the Main Belt, only $v_{6}$ and 3:1 resonances are efficient NEA sources. Some of the most important numerical results derived by Gladman et al. (1997), Morbidelli \& Gladman (1998) and Bottke et al. (2002) concerning the $v_{6}$, 3:1 and 5:2 resonances, are summarized in Table 1.

On the other hand, the dynamical structure of the $2: 1$ resonance is somewhat complicated. The numerical simulations developed by Gladman et al. (1997) suggest that the median lifetime of bodies initially in the 2:1 resonance is larger than
100 Myr. But, the work of Brož et al. (2005), which reexamines the origin, evolution and survivability of objects in the 2:1 population, suggests that the Yarkovsky effect (see next section) continuously resupplies bodies to this resonance and keeps the unstable population in an approximately steady state, obtaining lifetimes ranging from a few million years to $\sim 100 \mathrm{Myr}$ with a median lifetime of around $10 \mathrm{Myr}$. Thus, the 2:1 resonance is capable of perturbing the asteroid motion on timescales comparable to those of the other powerful resonances (see Table 1).

On the contrary, the diffusive resonances have no associated deep gaps in the Main Belt asteroid distribution. There are hundreds of these weak resonances that densely cross the Main Belt. They are represented by:

- high order mean motion resonances with Jupiter,

- three-body resonances with Jupiter and Saturn (Murray et al. 1998; Nesvorný \& Morbidelli 1998, 1999), and

- mean motion resonances with Mars (Morbidelli \& Nesvorný 1999).

The existence of these diffusive resonances leads many Main Belt asteroids to present a chaotic behavior (Nesvorný et al. 2002), even though the effect of this chaoticity results to be very weak. These thin resonances can produce slow changes of the orbital parameters of objects, leading them to evolve into planetcrossing orbits. Particularly in the inner ring of the asteroid belt, the diffusive resonances can explain the existence of one distinctive population of small bodies known as the Mars-crosser population (see Fig. 1). According to Migliorini et al. (1998), Mars-crossers are defined as those bodies with $q>1.3 \mathrm{AU}$ and a combination of $(a, e, i)$ values such that they cross the orbit of Mars during a secular oscillation cycle of their eccentricity. While the main population of Mars-crossers, called Intermediate Source Mars-Crossers (IMC), is situated below the $v_{6}$ resonance, there are other small groups with high inclination. Michel et al. (2000) developed numerical simulations of the dynamical evolution of objects on Mars-crossing orbits. These works show that asteroids belonging to IMC group can become NEAs over a time scale of several tens of millions of years. Later on, Bottke et al. (2002) integrated thousands of test particles from different NEA source regions in order to compute the orbital and absolute magnitude distribution of this population. The quantitative result of this work determines that IMC population must be considered as an important source of NEAs, together with the $v_{6}$ and $3: 1$ resonances.

In Table 2, we have summarized some of the most important dynamical results derived by Bottke et al. (2002) with regards to the different NEA source regions studied by them. It is possible to argue that the $v_{6}$ secular resonance, the intermediate-source Mars-crossing (IMC) population and the 3:1 mean motion resonances with Jupiter are the primary NEA sources, while the Outer Main Belt (OB) and the Jupiter-family comets (JFC) are only secondary sources.

\subsection{Yarkovsky effect}

The Yarkovsky effect is the result of a radiation mechanism which can cause relevant changes in the orbital parameters of the Solar System rotating bodies because of the asymmetry between the direction of absorption of sunlight and the direction of re-emission of thermal radiation. There are two variants of this mechanism for a rotating body moving around the Sun:

- the diurnal effect due to the rotation motion around its axis, - the seasonal effect due to the orbital motion. 
Table 2. Some of the most important dynamical results derived by Bottke et al. (2002) for bodies coming from the different NEA source regions. The sources studied by these authors were the $v_{6}$ and 3:1 resonances, the intermediate source Mars-crossing (IMC) population (namely, Marscrossers below the $\left.v_{6}\right)$, the Outer Main Belt $(\mathrm{OB})$ and the Jupiter-family comets. $N_{\mathrm{NEA}}(H<18)$ represents the steady-state number of NEAs with an $H$ magnitude smaller than 18, which is roughly $1 \mathrm{~km}$ in size. On the other hand, $\tau\left(\mathrm{Myr}^{-1}\right)$ gives the number of bodies injected into the NEA region per million years while $T_{\mathrm{NEA}}(\mathrm{Myr})$ is the mean dynamical lifetime spent in the NEA region.

\begin{tabular}{lcccccc}
\hline \hline & $v_{6}$ & IMC & $3: 1$ & OB & JFC & Total \\
\hline$N_{\text {NEA }}(H<18)$ & $360 \pm 90$ & $240 \pm 40$ & $220 \pm 90$ & $79 \pm 12$ & $61 \pm 43$ & $960 \pm 120$ \\
$\tau\left(\mathrm{Myr}^{-1}\right)$ & $55 \pm 18$ & $65 \pm 15$ & $100 \pm 50$ & $570 \pm 120$ & - & $790 \pm 200$ \\
$T_{\text {NEA }}(\mathrm{Myr})$ & 6.54 & 3.75 & 2.16 & 0.14 & - & - \\
\hline
\end{tabular}

The magnitude of both effects depends on the obliquity. While the diurnal effect vanishes at $90^{\circ}$ obliquity, the seasonal one vanishes at zero obliquity. Besides, the diurnal effect reaches its maximum value at zero obliquity while the seasonal one is maximum at $90^{\circ}$ obliquity. It is important to take into account that the seasonal effect always produces a semimajor axis decrease, while the diurnal can cause an increase when the rotation is prograde or a decrease when the rotation is retrograde.

On the other hand, the Yarkovsky effect is size dependent. Actually, this mechanism affects the orbital parameters of small asteroids, under the kilometer size range, while large asteroids are mostly unaffected. Because of this dependence with the size, the Yarkovsky effect can potentially affect the Main Belt size distribution. Besides, it is important to take into account that since this mechanism is the result of a radiation force, its efficiency drops with increasing semimajor axis from the Sun.

Several interesting works were developed in the last decade in order to study this radiation mechanism. Farinella et al. (1998) derived a unified model of the Yarkovsky effect in both the diurnal effect and also for the seasonal one, obtaining explicit expressions for the semimajor axis drift rates. Penco et al. (2002) included the Yarkovsky effect into the numerical models of the collisional evolution of the asteroid Main Belt. Later, Morbidelli \& Vokrouhlický (2003) developed simulations in order to study the role of the Yarkovsky effect in the origin of near-Earth asteroids. This work argues that the Yarkovsky effect is the major mechanism by which asteroids are continuously supplied to powerful and diffusive resonances and the NEA population is maintained in steady state. These conclusions lead us to think that this radiation mechanism together with the resonant escape routes can be an important source of steady mass depletion in the Main Belt.

There are several mechanisms that can modify the effectiveness of the Yarkovsky effect. In fact, the Yarkovsky-O'KeefeRadzievskii-Paddack effect, or YORP effect, and the collisional re-orientations of the spin axes of the asteroids can produce changes of the obliquity states of such objects, leading them to random walk in semimajor axis rather than a continuous drift. Moreover, the detailed numerical simulations performed by Morbidelli \& Vokrouhlický (2003) show the importance of the YORP effect for understanding why the NEA magnitude distribution is only moderately steeper than the Main Belt magnitude distribution.

In order to quantify the removal rate of bodies due to the action of these radiation forces and orbital resonances, Sect. 4.4 shows a simplified mathematical description of these mechanisms. Later, Sect. 4.5 describes how these dynamical processes can be included in our numerical algorithm.

\section{Collisional and dynamical evolution model}

In this section we present the full model we use to study the simultaneous evolution of the asteroid Main Belt and NEA population.

\subsection{Populations of the model}

As we have already said, the most important resonances of the powerful class are the $v_{6}$ secular resonance and the mean motion resonances with Jupiter 3:1, 5:2 and 2:1 at about 2.5, 2.8 and 3.27 AU from the Sun, respectively. Taking into account that less than 1 percent of the Main Belt population is located between 3.27 and 3.4 AU, we have decided to assume that 2:1 resonance marks the outer edge of the asteroid belt. In order to study the flux of asteroids into these resonances as a result of the collisional injection and the Yarkovsky effect, and analyze the mixing of material between the different regions of the asteroid belt, our model divides the Main Belt into three semimajor axis zones:

- the Inner Ring (IR), between the $v_{6}$ and 3:1 resonances, from about 2 to $2.5 \mathrm{AU}$,

- the Middle Ring (MR), between the 3:1 and 5:2 resonances, from about 2.5 to $2.823 \mathrm{AU}$, and

- the Outer Ring (OR), between the 5:2 and 2:1 resonances, from about 2.823 to $3.27 \mathrm{AU}$.

The width of every ring is determined by the resonance borders. The boundaries of the 3:1 and 5:2 resonances depend on the orbital eccentricity while the semimajor axis of the center of the $v_{6}$ secular resonance depends on the orbital inclination, but only weakly on the eccentricity (Morbidelli \& Henrard 1991). To define the boundaries of the $v_{6}$ and 3:1 resonances, we follow Morbidelli \& Vokrouhlický (2003). They have shown that the boundaries can be approximated by

$$
\begin{aligned}
& a=2.508+\frac{e}{29.615} \quad \text { for } \quad e \leq 0.15936 \text {, } \\
& a=2.485+\frac{e}{5.615} \quad \text { for } \quad e>0.15936
\end{aligned}
$$

for the right side of the 3:1 resonance,

$$
\begin{aligned}
& a=2.492-\frac{e}{108.85} \quad \text { for } \quad e \leq 0.1734 \\
& a=2.51-\frac{e}{8.85} \quad \text { for } \quad e>0.1734
\end{aligned}
$$

for the left side of the $3: 1$ resonance, and

$a=2.12+6.003(\sin i)^{2.256}$,

for the right side of the $v_{6}$ resonance. In order to take into account the diffusive neighborhood of these resonances to correctly 

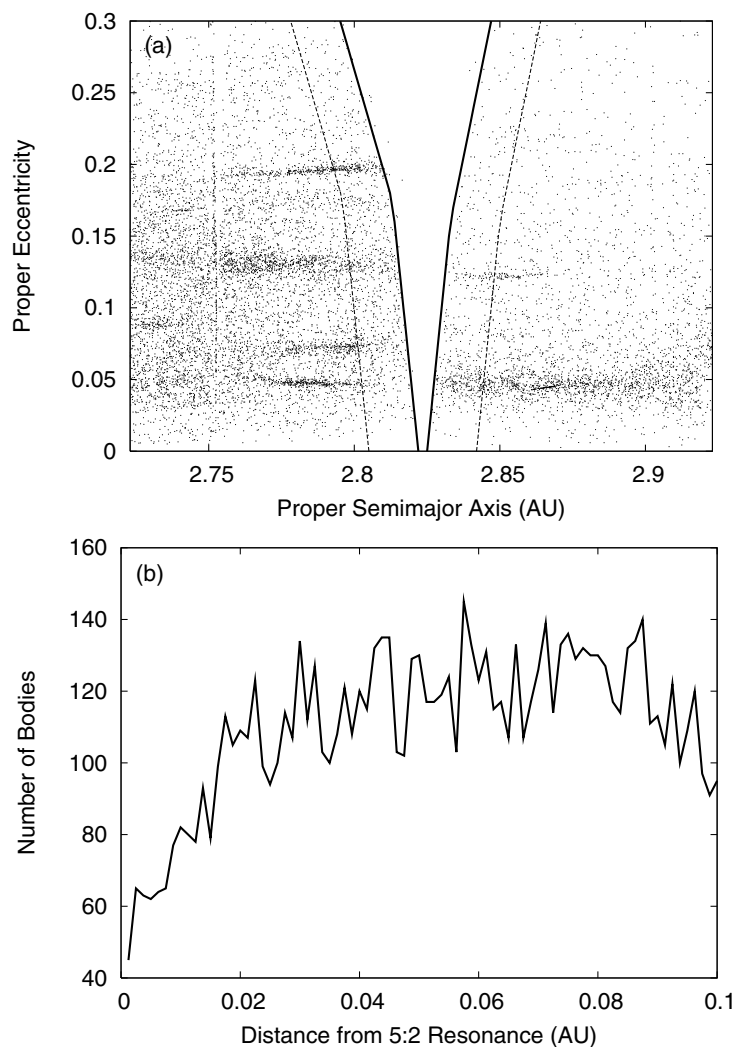

Fig. 2. a) The distribution of the asteroids with respect to proper semimajor axis and eccentricity, in the vicinity of the 5:2 mean motion resonance with Jupiter. The right and left boundaries of this resonance approximated by Eqs. (32) and (33) respectively, are represented by the solid curves. The shifted boundaries defined in order to evaluate the effective flux of asteroids into this resonant region are labeled by the dashed curves. b) The number of asteroids as a function of distance from the left border of the 5:2 resonance. The curve shows that the density of objects grows until $\sim 0.017 \mathrm{AU}$ from the resonance, remaining more or less constant over the next $0.03 \mathrm{AU}$.

evaluate the effective flux of asteroids into them, Morbidelli \& Vokrouhlický (2003) shifted the boundaries of the 3:1 resonance by $0.015 \mathrm{AU}$ away from the borders given by Eqs. (29) and (30), while they also drifted the $v_{6}$ resonance boundary, given by Eq. (31), outward by 0.09 AU.

To define the effective boundaries of the 5:2 resonance, we performed a similar analysis. Using the catalog of the synthetic proper elements (Knežević \& Milani 2003), it is possible to see a well-defined gap associated with the 5:2 resonance, which is illustrated in Fig. 2a. The boundaries of this resonance can be approximated as

$\begin{array}{llll}a=2.825+\frac{e}{20} & \text { for } & e \leq 0.16, \\ a=2.817+\frac{e}{10} & \text { for } & e>0.16,\end{array}$

for the right side, and

$\begin{array}{llrl}a & =2.822-\frac{e}{19.5} \quad \text { for } & e \leq 0.17, \\ a=2.838-\frac{e}{7} & \text { for } & e>0.17,\end{array}$

for the left side. We must extend the boundaries of the 5:2 resonance because of the chaotic diffusion in the vicinity of its borders. We observed that the density of asteroids grows until
Table 3. Mean values for the intrinsic collision probability $\left\langle P i_{\mathrm{c}}\right\rangle$ and the impact velocity $\langle V\rangle$ for the different populations of our model.

\begin{tabular}{|c|c|c|}
\hline 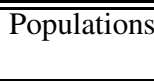 & $\begin{array}{c}\left\langle P i_{\mathrm{c}}\right\rangle \\
\left(10^{-18} \mathrm{~km}^{-2} \mathrm{yr}^{-1}\right) \\
\end{array}$ & $\begin{array}{c}\langle V\rangle \\
\left(\mathrm{km} \mathrm{s}^{-1}\right) \\
\end{array}$ \\
\hline & Main Belt-Main Bel & \\
\hline IR-IR & 9.8 & 3.7 \\
\hline IR-MR & 8.6 & 4.4 \\
\hline IR-OR & 1.4 & 4.4 \\
\hline MR-MR & 1.7 & 4.3 \\
\hline MR-OR & 3.5 & 4 \\
\hline OR-OR & 2.74 & 3.3 \\
\hline \multicolumn{3}{|c|}{ Main Belt-NEA } \\
\hline IR-NEA & 6.2 & 9.9 \\
\hline MR-NEA & 2.7 & 9.3 \\
\hline OR-NEA & 1.5 & 8.1 \\
\hline \multicolumn{3}{|c|}{ NEA-NEA } \\
\hline NEA-NEA & 7.2 & 14.5 \\
\hline
\end{tabular}

$\sim 0.015-0.017 \mathrm{AU}$ from the resonance and then is more or less constant over the next $0.03 \mathrm{AU}$ (see Fig. 2b). In order to measure the effective flux of asteroids falling into the 5:2 resonance, we shift the boundaries of this region $0.017 \mathrm{AU}$ away from the borders given by the Eqs. (32) and (33).

In the following, these shifted boundaries will be the boundaries of the $v_{6}, 3: 1$ and 5:2 resonances with which we are going to develop our work.

\subsection{Collision velocities and probabilities}

Mean values for the impact velocity $\langle V\rangle$ and the intrinsic collision probability $\left\langle P i_{\mathrm{c}}\right\rangle$, are fundamental quantities for any collisional evolution study. We calculate $\langle V\rangle$ and $\left\langle P i_{\mathrm{c}}\right\rangle$ for collisions between asteroids of the Main Belt in every ring and between rings as well as for collisions between NEAs and between NEAs and Main Belt objects in every ring. For this, we use the numerical approach developed by Marzari et al. (1996), based in the numerical integration of 3000 real asteroids from the three populations, subject to the perturbations of Jupiter and Saturn. The timespan of the numerical integration was of $10^{4} \mathrm{yr}$, and the integration was performed with the simplectic code EVORB (Fernández et al. 2002). The results are shown in Table 3.

\subsection{Asteroid strength}

O'Brien \& Greenberg (2005) showed that the general shape of the final evolved asteroid population is determined primarily by $Q_{\mathrm{D}}$, but variations in $Q_{\mathrm{S}}$ and $f_{\mathrm{ke}}$ can affect such final population even if $Q_{\mathrm{D}}$ is held the same. According to these arguments we choose $Q_{\mathrm{S}}$ and $f_{\mathrm{ke}}$ as input parameters of our collisional model.

The $Q_{\mathrm{S}}$ law chosen from this study is shown in Fig. 3a, which can be calculated from an expression of the form

$Q_{\mathrm{S}}=C_{1} D^{-\lambda_{1}}\left(1+\left(C_{2} D\right)^{\lambda_{2}}\right)$

where $C_{1}, C_{2}, \lambda_{1}$, and $\lambda_{2}$ are constant coefficients whose values are $2.85,1.8,0.695$ and 2.22 , respectively. As the reader can see in Fig. 3a, this $Q_{\mathrm{S}}$ law is in a good agreement with the estimates of the impact strength of asteroids proposed by different authors (Farinella et al. 1982; Davis et al. 1985; Housen \& Holsapple 1990; Housen 1991; Holsapple 1994; Ryan \& Melosh 1998; Benz \& Asphaug 1999).

On the other hand, $f_{\mathrm{ke}}$ is a poorly known parameter in collisional processes. But, many authors suggest that it may vary with 

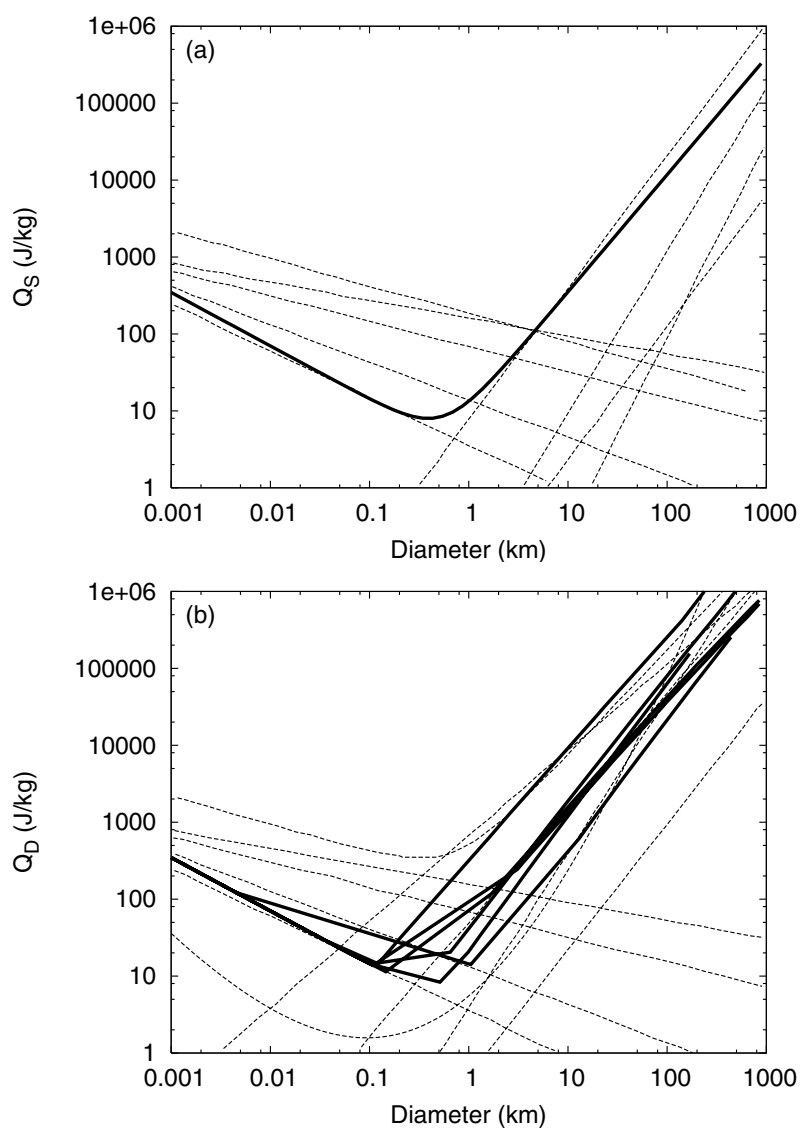

Fig. 3. a) Asteroid strength laws $Q_{\mathrm{S}}$. The solid curve denotes the $Q_{\mathrm{S}}$ law used in our work. The dashed lines represent the estimates proposed by different authors. b) Asteroid strength laws $Q_{\mathrm{D}}$. The solid curves denote the $Q_{\mathrm{D}}$ laws generated from our $Q_{\mathrm{S}}$ law and the different functions $f_{\mathrm{ke}}$ used in the populations of the model. The dashed lines represent the estimates proposed by different authors.

size, with impact speed and probably with the material properties. Since the impact velocity varies for the different populations of our model (see Table 3 ) and the heliocentric distribution of taxonomic classes (Mothé-Diniz et al. 2003) indicates differences in the asteroid composition of the Main Belt, we have decided that $f_{\mathrm{ke}}$ varies for the different populations. Thus, according to that made by O'Brien \& Greenberg (2005), we express the parameter $f_{\mathrm{ke}}$ as

$f_{\mathrm{ke}}=f_{\mathrm{ke}_{0}}\left(\frac{D}{1000 \mathrm{~km}}\right)^{\gamma}$.

Table 4 shows the values of $f_{\mathrm{ke}_{0}}$ and $\gamma$ for the different populations of our model. Such values are according to that discussed by O'Brien \& Greenberg (2005), who indicate that $\gamma$ is on the order of 0.5 (always between 0 and 1) and $f_{\mathrm{ke}_{0}}$, the value at $1000 \mathrm{~km}$, is $\sim 0.05-0.3$, which is consistent with estimates of $f_{\mathrm{ke}}$ in large impacts (Davis et al. 1989). It is important to take into account that the combination of $Q_{\mathrm{S}}$ and $f_{\mathrm{ke}}$ yields a given $Q_{\mathrm{D}}$. The $Q_{\mathrm{D}}$ laws generated from our $Q_{\mathrm{S}}$ law and the different functions $f_{\mathrm{ke}}$ are shown in Fig. $3 \mathrm{~b}$. Such $Q_{\mathrm{D}}$ laws are in a good agreement with those formulated by different authors (Farinella et al. 1982; Davis et al. 1985; Housen \& Holsapple 1990; Holsapple 1994; Love \& Ahrens 1996; Melosh \& Ryan 1997; Durda et al. 1998; Ryan \& Melosh 1998; Benz \& Asphaug 1999), and are according to the laboratory impact experiments which obtain values near $1500 \mathrm{~J} \mathrm{~kg}^{-1}$ for target diameters of $\sim 8 \mathrm{~cm}$.
Table 4. Values for $f_{\mathrm{ke}_{0}}$ and $\gamma$ for the different populations of our model.

\begin{tabular}{lcc}
\hline \hline Populations & $f_{\mathrm{ke}_{0}}$ & $\gamma$ \\
\hline \multirow{3}{*}{ IR-IR } & Main Belt-Main Belt & \\
IR-MR & 0.05 & 0.5 \\
IR-OR & 0.1 & 0.2 \\
MR-MR & 0.1 & 0.3 \\
MR-OR & 0.3 & 0.5 \\
OR-OR & 0.3 & 0.2 \\
\hline & 0.35 & 0.5 \\
IR-NEA & Main Belt-NEA \\
MR-NEA & 0.2 & 0.4 \\
OR-NEA & 0.2 & 0.4 \\
\hline & 0.2 & 0.4 \\
NEA-NEA & NEA-NEA \\
\hline
\end{tabular}

\subsection{Asteroid removal due to the Yarkovsky effect and orbital resonances}

In order to calculate the removal rate of bodies from each of the three rings of the asteroid Main Belt due to the action of the Yarkovsky effect and orbital resonances, we use the expressions derived by O'Brien \& Greenberg (2005), which are based on the analytical model outlined by Farinella et al. (1998). Here, we give a brief mathematical description of this effect, separately considering the treatments developed for the diurnal and seasonal variants of this radiation mechanism (Sect. 3.2).

The diurnal variant is the simplest case of the Yarkovsky effect. This variant is due to the fact that a rotating body absorbing radiation from the Sun rotates before that energy is re-emitted as thermal infrared radiation, leading to a longitudinal asymmetry between the direction of absorption of sunlight and the direction of re-emission. The discussion presented in Farinella et al. (1998) suggests that the diurnal Yarkovsky effect is effective for all bodies larger than about 3 microns while, for smaller bodies, its effectiveness does not become important since the heating on one side of the object begins to affect the other side.

Following Farinella et al. (1998), if $F_{\mathrm{Y}}$ is the along-track component of the Yarkovsky force per unit mass of the body, the semimajor axis change is given by

$\dot{a}=\frac{2 F_{\mathrm{Y}}}{n}$,

where $n=2 \pi / P_{\text {orb }}$ is the orbital mean motion and, according to Burns et al. (1979), $F_{\mathrm{Y}}$ can be expressed by the formula

$F_{\mathrm{Y}}=\frac{2}{\rho R} \frac{\epsilon \sigma T^{4}}{c} \frac{\Delta T}{T} f(\zeta)$

where $\rho$ is the density of the body (assumed to be $3500 \mathrm{~kg} \mathrm{~m}^{-3}$, which is the density for basalt), $R$ is its radius, $\epsilon$ is the surface infrared emissivity (assumed to be 1), $\sigma$ is the Stefan-Boltzmann constant, $c$ is the speed of light, $T$ is the effective temperature of the body, $\Delta T$ is the effective temperature difference and $f(\zeta)$ is the obliquity function. The effective temperature of the body can be calculated equating the incoming solar flux to the radiated flux from the asteroid. From this,

$\pi R^{2}(1-A) S=4 \pi R^{2} \epsilon \sigma T^{4}$,

where $A$ is the albedo (assumed to be zero) and $S$ is the solar flux in the position of the body. $S$ depends on the semimajor axis and can be expressed by the formula

$S=S_{0}\left(\frac{a_{0}}{a}\right)^{2}$ 

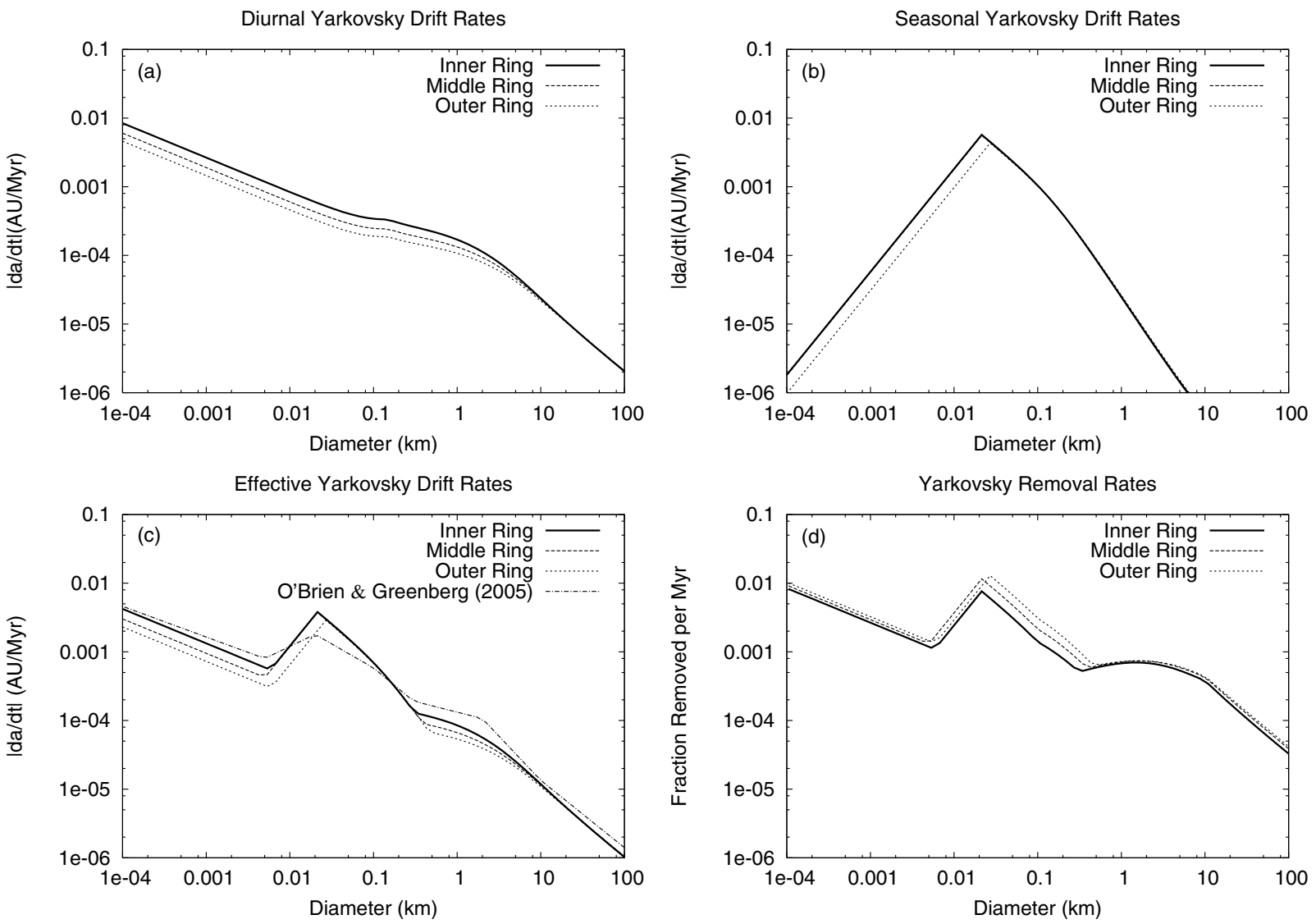

Fig. 4. Our estimates of the diurnal a), seasonal b) and effective c) Yarkovsky semimajor axis drift rates $\dot{a}$ and the asteroid removal rates d) as a function of the diameter for bodies belonging to the Inner, Middle and Outer Rings of the asteroid Main Belt.

where $S_{0}=1370 \mathrm{~W} \mathrm{~m}^{-2}$ is the solar constant and $a_{0}$ and $a$ are the semimajor axes of the Earth and the body under consideration, respectively. Finally, the average temperature gives

$T=\left\{\frac{(1-A) S}{4 \epsilon \sigma}\right\}^{1 / 4}$

The effective temperature difference $\Delta T$ and the obliquity function $f(\zeta)$ adopt different expressions in the diurnal and seasonal variants of this mechanism. From Peterson (1976), for the diurnal effect we have $f_{\mathrm{d}}(\zeta)=\cos \zeta$ and $\Delta T_{\mathrm{d}}$ can be calculated by the formula

$\frac{\Delta T_{\mathrm{d}}}{T}=\frac{2}{3} \frac{\Theta_{\mathrm{d}}}{1+2.03 \Theta_{\mathrm{d}}+2.04 \Theta_{\mathrm{d}}^{2}}$

$\Theta_{\mathrm{d}}$ is a thermal parameter defined by Farinella et al. (1998) and represents the ratio of the thermal emission timescale to the rotation timescale. This parameter is given by

$\Theta_{\mathrm{d}}=\frac{\sqrt{\rho C K}}{2 \pi \epsilon \sigma T^{3}} \sqrt{\omega}$,

where $K$ is the thermal conductivity, $\rho$ is the density, $C$ is the specific heat (assumed to be $680 \mathrm{~J} \mathrm{~kg}^{-1} \mathrm{~K}^{-1}$, which is the value corresponding to basalt and regolith) and $\omega=2 \pi / P_{\text {rot }}$ is the rotation frequency. According to O'Brien \& Greenberg (2005), we use $P_{\text {rot }}=6 \mathrm{~h}$ for bodies larger than $0.15 \mathrm{~km}$ and $P_{\text {rot }} \propto D$ (Farinella et al. 1998) for smaller bodies. In same way, we model the density $\rho$ and the thermal conductivity $K$ for bodies smaller than $0.15 \mathrm{~km}$ using basalt parameters $\left(\rho_{\text {rock }}=3500 \mathrm{~kg} \mathrm{~m}^{-3}\right.$ and $K_{\text {rock }}=2.65 \mathrm{~W} \mathrm{~m}^{-1} \mathrm{~K}^{-1}$ ) while for larger bodies, we use regolith parameters $\left(\rho_{\text {reg }}=1500 \mathrm{~kg} \mathrm{~m}^{-3}\right.$ and $\left.K_{\text {reg }}=0.0015 \mathrm{~W} \mathrm{~m}^{-1} \mathrm{~K}^{-1}\right)$, with a smooth variation between them around $0.15 \mathrm{~km}$ ( $\mathrm{O}^{\prime}$ Brien $\&$ Greenberg 2005). Figure $4 \mathrm{a}$ shows the semimajor axis mobility due to the diurnal Yarkovsky effect as a function of the diameter for bodies belonging to the Inner, Middle and Outer Rings of the asteroid Main Belt. The obliquity is assumed to be $0^{\circ}$ in order to consider its maximum effect (Sect. 3.2).

On the other hand, the seasonal Yarkovsky effect is due to the fact that a body absorbing radiation from the Sun moves in its orbit before that energy is re-emitted as thermal infrared radiation, leading to a latitudinal asymmetry between the direction of absorption of sunlight and the direction of re-emission. The mathematical description of the seasonal Yarkovsky effect is somewhat more complicated. However, from O'Brien \& Greenberg (2005), we approximate the seasonal effect considering it like a diurnal one with frequency $n$ (orbital mean motion) rather than $w$ (rotation frequency), assuming that for this variant $f_{\mathrm{s}}(\zeta)=-\sin ^{2} \zeta$, and knowing that the seasonal asymmetry must be taken into account for only a fraction of the orbit. Thus, the effective temperature difference $\Delta T_{\mathrm{s}}$ can be calculated by the expression

$\frac{\Delta T_{\mathrm{s}}}{T}=\frac{2}{3} \frac{\Theta_{\mathrm{s}}}{1+2.03 \Theta_{\mathrm{s}}+2.04 \Theta_{\mathrm{s}}^{2}} f_{a}$,

where the thermal parameter $\Theta_{\mathrm{s}}$ is given by

$\Theta_{\mathrm{s}}=\frac{\sqrt{\rho C K}}{2 \pi \epsilon \sigma T^{3}} \sqrt{n}$,

and the factor $f_{a}$ (assumed to be $2 / \pi$ ) takes into account the partial asymmetry. The work developed by Farinella et al. (1998) 
indicates that the peak seasonal Yarkovsky effect occurs at diameters of about $20 \mathrm{~m}$. To calculate the seasonal force for smaller bodies, it is necessary a mathematical description somewhat more complicated. According to O'Brien \& Greenberg (2005), we model the seasonal Yarkovsky effect for bodies smaller than $20 \mathrm{~m}$ in diameter assuming that $\dot{a}_{\mathrm{s}} \sim D^{3 / 2}$, which is in agreement with that made by Farinella et al. (1998). Figure 4b shows the semimajor axis mobility due to the seasonal Yarkovsky effect as a function of the diameter for bodies belonging to the Inner, Middle and Outer Rings of the asteroid Main Belt. The obliquity is assumed to be $90^{\circ}$ in order to consider its maximum effect (Sect. 3.2).

The effective Yarkovsky semimajor axis drift rate for each of the three rings of the Main Belt is a combination for the seasonal and diurnal effects. Taking into account that the mean absolute values of the obliquity functions $f_{\mathrm{d}}(\zeta)$ and $f_{\mathrm{s}}(\zeta)$ are $1 / 2$ and $2 / 3$, respectively, so the maximum and minimum absolute values of effective $\dot{a}$ are given by

$$
\begin{aligned}
|\dot{a}(D)|_{\min }= & \operatorname{abs}\left(\left\langle\left|f_{\mathrm{s}}(\zeta)\right|\right\rangle\left|\dot{a}_{\mathrm{s}}\left(D, \zeta=90^{\circ}\right)\right|\right. \\
& \left.-\left\langle\left|f_{\mathrm{d}}(\zeta)\right|\right\rangle\left|\dot{a}_{\mathrm{d}}\left(D, \zeta=0^{\circ}\right)\right|\right) \\
|\dot{a}(D)|_{\max }= & \operatorname{abs}\left(\left\langle\left|f_{\mathrm{s}}(\zeta)\right|\right\rangle\left|\dot{a}_{\mathrm{s}}\left(D, \zeta=90^{\circ}\right)\right|\right. \\
& \left.+\left\langle\left|f_{\mathrm{d}}(\zeta)\right|\right\rangle\left|\dot{a}_{\mathrm{d}}\left(D, \zeta=0^{\circ}\right)\right|\right),
\end{aligned}
$$

and then, the average absolute value of effective $\dot{a}$ will be

$\langle\dot{a}(D)\rangle=\frac{\left.|\dot{a}(D)|\right|_{\min }+|\dot{a}(D)|_{\max }}{2}$,

(O'Brien \& Greenberg 2005). Figure 4c shows the effective Yarkovsky semimajor axis drift rates $\dot{a}$ as a function of the diameter for bodies belonging to the Inner, Middle and Outer Rings of the asteroid Main Belt, which are consistent with that obtained by O'Brien \& Greenberg (2005) for the entire Main Belt.

Once $\langle\dot{a}(D)\rangle$ has been determined, the fraction of bodies of diameter $D$ removed per unit time can be calculated from the expression

$f_{\text {rem }}(D)=\frac{\langle\dot{a}(D)\rangle}{\Delta a} N_{\text {res }}$,

where $\langle\dot{a}(D)\rangle$ is the effective Yarkovsky semimajor axis drift rate, $\Delta a$ is the effective width of the considered region and $N_{\text {res }}$ is the number of resonances that can remove bodies of a given diameter $D$ from such region. The fraction of bodies removed per unit time is not linearly proportional to $\langle\dot{a}(D)\rangle$ since $N_{\text {res }}$ depends on the diameter $D$. In fact, small bodies $(D \lesssim 0.1 \mathrm{~km})$ have high Yarkovsky drift rates and owing to that they can jump weak resonances, being only removed by powerful resonances. Following O'Brien \& Greenberg (2005), we assume that the weak resonances start to be effective for bodies around $0.1 \mathrm{~km}$ while all of them become fully effective for bodies $10 \mathrm{~km}$ in diameter or larger. Thus, we assume that $N_{\text {res }}$ will be equal to the number of powerful resonances in the considered region at $D \leq 0.1 \mathrm{~km}$ while $N_{\text {res }}$ will be equal to the number of powerful resonances plus the approximate number of diffusive resonances at $D \geq$ $10 \mathrm{~km}$, considering a linear variation between them for intermediate diameters. To evaluate the number of strong resonances in each ring, we take into account the possible escape routes from such regions. $\Delta a /\langle\dot{a}(D)\rangle$ represents an estimate of the median lifetime of a body of diameter $D$ in a region with an effective width $\Delta a$, where the escape routes are located at the borders. Since the Inner and Middle Rings of the asteroid belt are divided by the $v_{6}, 3: 1$ and 5:2 powerful resonances and besides, we consider that there are no other strong resonances inside such regions, the number of powerful resonances is assumed to be 1 for the Inner and Middle Rings. For the Outer Ring, whose boundaries are given by the 5:2 and $2: 1$ powerful resonances, we also consider the existence of the $7: 3$ strong resonance at $\sim 2.96 \mathrm{AU}$, and owing to that the number of powerful resonances is assumed to be 2 for the Outer Ring. On the other hand, we consider 16, 12 and 18 diffusive resonances for the Inner, Middle and Outer Rings of the asteroid belt, respectively. It is important to take into account that our model of the Yarkovsky effect and the number of the orbital resonances associated with each ring of the Main Belt must be consistent with the results obtained by Morbidelli \& Nesvorný (1999) and Bottke et al. (2002) with regard to the dynamical removal rate for multi-kilometer bodies. In fact, while Morbidelli \& Nesvorný (1999) estimate the escape of about 4 bodies larger than $5 \mathrm{~km}$ per million years from the Inner Belt, Bottke et al. (2002) indicate that $790 \pm 200$ bodies larger than $1 \mathrm{~km}$ are removed from the entire Main Belt per million years. The Yarkovsky asteroid removal rates obtained from this analysis for each of the three rings of the asteroid Main Belt are shown in Fig. 4d.

But, as O'Brien \& Greenberg (2005), we do not take into account the YORP effect nor collisional re-orientations of the spin axes and owing to that, it is likely that the real removal rates of asteroids from the different regions of the Main Belt differ somewhat from our estimates, but they probably show similar trends and are of the same order of magnitude. So, since the asteroid removal rates obtained from our analysis are only an estimate, we decide to slightly vary them in order to obtain better fits to the observed populations of the model and results consistent with those found by Morbidelli \& Nesvorný (1999) and Bottke et al. (2002) with regard to the dynamical removal rate for multi-kilometer bodies. Figure 5 shows the asteroid removal rates used in our algorithm.

\subsection{The full model}

In order to simulate the collisional and dynamical evolution of the asteroid Main Belt and NEA size distributions, our numerical code evolves in time the number of bodies associated with each of the three rings of the asteroid belt and NEA population. The populations of objects reside in a set of 130 discrete logarithmic size bins, whose central values range from $D_{1}=10^{-10} \mathrm{~km}$ to $D_{130}=886.7 \mathrm{~km}$ in diameter in such a way that from one bin to the next, the mass of the bodies changes by a factor of 2 and the diameter changes by a factor of $2^{1 / 3}$. We adopt a density of $2.7 \mathrm{~g} \mathrm{~cm}^{-3}$.

While the NEA population always starts with zero bodies, the total mass of the objects associated with each of the three rings of the asteroid Main Belt is calculated from the model of planetary nebulae mass distribution proposed by Weidenschilling (1977). We adopt a surface density $\Sigma$ of the nebular disk of the form

$\Sigma(a)=\Sigma_{0}\left(\frac{a}{a_{0}}\right)^{-3 / 2}$,

where $\Sigma_{0}$ is the value associated to an arbitrary radius $a_{0}$. Thus, the differential mass $\mathrm{d} M(a)$ contained in a belt of radius $a$ and width $\mathrm{d} a$ will be given by

$$
\begin{aligned}
\mathrm{d} M(a) & =2 \pi a \Sigma(a) \mathrm{d} a \\
& =2 \pi a \Sigma_{0}\left(\frac{a}{a_{0}}\right)^{-3 / 2} \mathrm{~d} a
\end{aligned}
$$



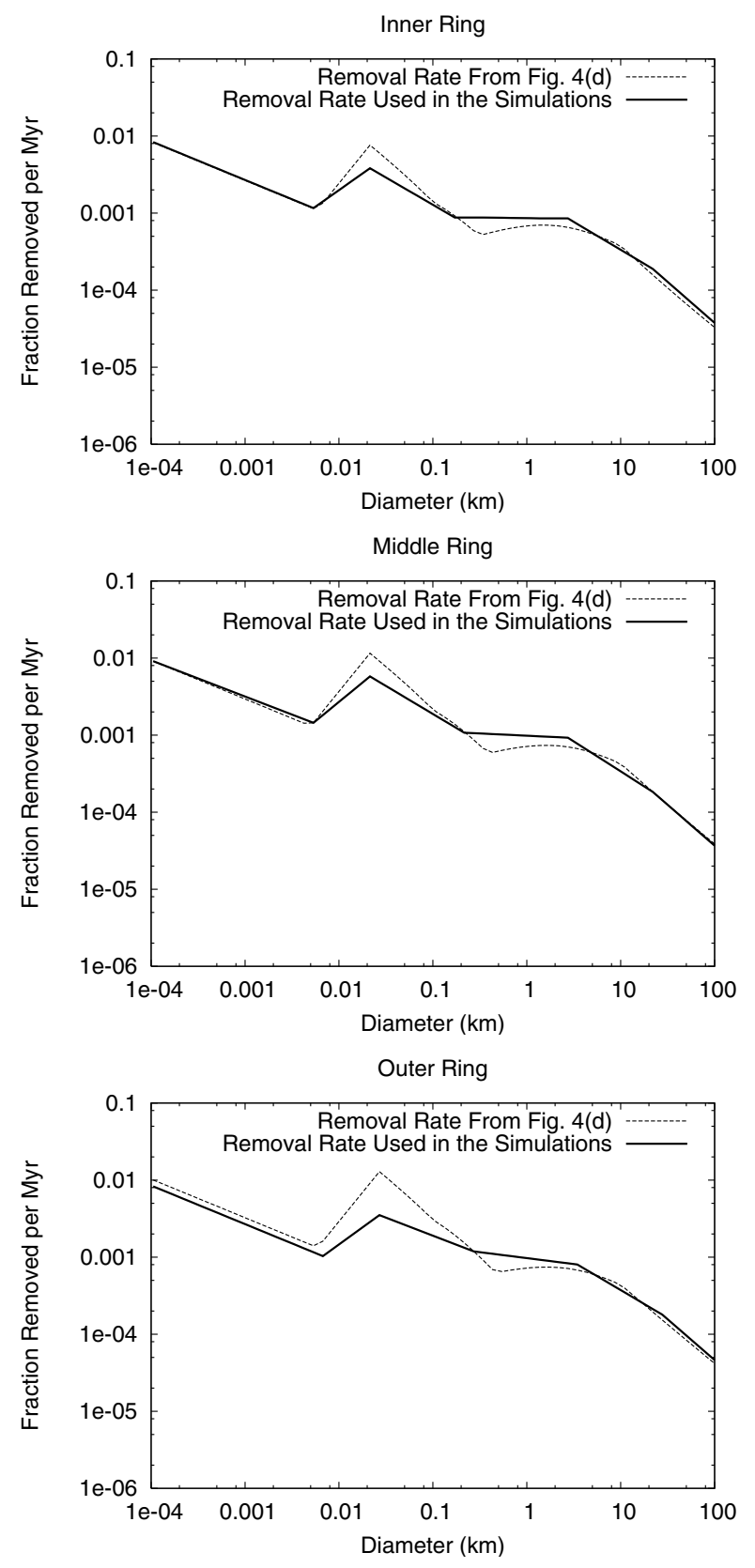

Fig. 5. Asteroid removal rates used in our simulation, compared to the estimates shown in Fig. 4d.

and from this, the mass of the entire Main Belt will be written as

$$
\begin{aligned}
M_{\mathrm{MB}} & =2 \pi \Sigma_{0} a_{0}^{3 / 2} \int_{2}^{3.27} a^{-1 / 2} \mathrm{~d} a \\
& =M_{0} \int_{2}^{3.27} a^{-1 / 2} \mathrm{~d} a .
\end{aligned}
$$

In the same way, the masses associated with each of the three rings of the asteroid Main Belt will be given by

$$
\begin{aligned}
& M_{\mathrm{IR}}=M_{0} \int_{2}^{2.5} a^{-1 / 2} \mathrm{~d} a \\
& M_{\mathrm{MR}}=M_{0} \int_{2.5}^{2.823} a^{-1 / 2} \mathrm{~d} a \\
& M_{\mathrm{OR}}=M_{0} \int_{2.823}^{3.27} a^{-1 / 2} \mathrm{~d} a .
\end{aligned}
$$

Proposing a given mass for the initial Main Belt, it is possible to obtain the constant $M_{0}$ from Eq. (50) and then to determine the corresponding masses for the Inner, Middle and Outer Rings, given by Eqs. (51). While we tested different initial masses, the results shown here are those obtained considering an initial belt with $\sim 7$ times the current belt mass, namely, 0.00315 Earth masses.

Once the masses associated with the Inner, Middle and Outer Rings of the asteroid Main Belt are determined, the next step is to construct the starting populations for each of these rings, which are defined as follow:

- Inner Ring ( $2<a<2.5 \mathrm{AU})$ : for $D \gtrsim 150 \mathrm{~km}$, we assume that the number of objects is equal to the observed number of asteroids in this region of the Main Belt. For $80 \lesssim D \lesssim$ $150 \mathrm{~km}$, we use an incremental power-law index of $\sim-1.6$, while for $D \lesssim 80 \mathrm{~km}$, the initial incremental population follows a slope of $\sim-3.1$ (see Fig. 6a).

- Middle Ring $(2.5<a<2.823 \mathrm{AU})$ : for $D \gtrsim 250 \mathrm{~km}$, we assume that the number of objects is equal to the observed number of asteroids in this region of the Main Belt. For $100 \lesssim D \lesssim 250 \mathrm{~km}$, we use an incremental power-law index of $\sim-2.2$, while for $D \lesssim 100 \mathrm{~km}$, the initial incremental population follows a slope of $\sim-2.9$ (see Fig. $6 b$ ).

- Outer Ring $(2.823<a<3.27 \mathrm{AU})$ : for $D \gtrsim 350 \mathrm{~km}$, we assume that the number of objects is equal to the observed number of asteroids in this region of the Main Belt. For $120 \lesssim D \lesssim 350 \mathrm{~km}$, we use an incremental power-law index of $\sim-3.3$, while for $D \lesssim 120 \mathrm{~km}$, the initial incremental population follows a slope of $\sim-2.9$ (see Fig. $6 \mathrm{c}$ ).

We construct these initial populations following the idea proposed by Bottke et al. (2005a) for the entire Main Belt. In fact, these authors use approximately the same number of objects as the observed Main Belt asteroids for $D>D_{1}=200 \mathrm{~km}$, while for $D_{2}<D<D_{1}=200 \mathrm{~km}$, where $D_{2}$ ranges around $100 \mathrm{~km}$, the population follows an incremental power-law index with a value close to the observed slope of asteroids in this size range (see Fig. 6). Here, we assume values for $D_{1}$ and $D_{2}$ of 150 and $80 \mathrm{~km}$, 250 and $100 \mathrm{~km}$, and 350 and $120 \mathrm{~km}$ for the Inner, Middle and Outer Rings, respectively. Then, for $D<D_{2}$, we assign a given incremental power-law index for every of the three rings of the Main Belt in order to reproduce their associated masses. From the combination of these three populations, the initial population associated with the entire Main Belt is determined. In fact, for $D \gtrsim 350 \mathrm{~km}$, the resulting initial population of the entire asteroid belt shows the same number of objects as the observed number of asteroids of the Main Belt. Moreover, for $200 \lesssim D \lessgtr$ $350 \mathrm{~km}$, the resulting population follows an incremental powerlaw index of $\sim-5$, while for $D \lesssim 200 \mathrm{~km}$, we assume a slope of $\sim-2.9$ (see Fig. 6d). These initial populations are consistent with the work of Bottke et al. (2005a) which indicates that it is not possible to reproduce the various waves of the asteroid Main Belt population assuming an unique power law at the beginning. On the other hand, the numerical simulations performed by Petit et al. (1999) and Petit et al. (2001) suggest that the asteroid Main Belt may have originally contained hundreds of times more mass that it currently has. Moreover, these authors indicate that the gravitational perturbations from Jupiter and primordial planetary embryos reduced very fast the mass of the initial belt, reaching its actual value over time scales of a few Myr. To take into account this result and following O'Brien \& Greenberg (2005), the initial populations associated with each of the three rings of the asteroid belt are initially multiplied by a given factor and their evolution followed for $5 \mathrm{Myr}$. Then, the residual populations are 

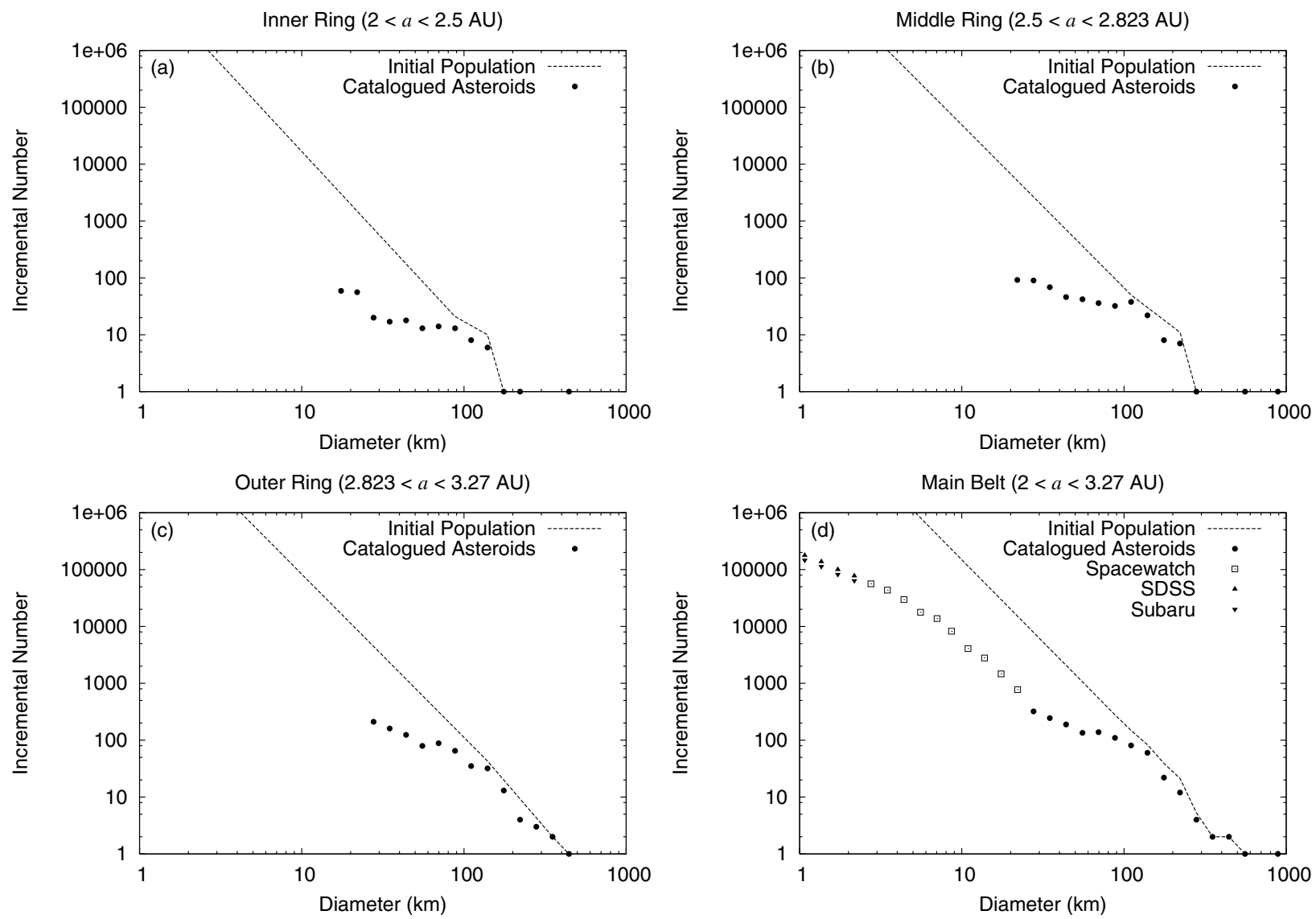

Fig. 6. Initial populations of the model.

reduced by the same factor and finally their evolution is analyzed for the rest of the $4.5 \mathrm{Gyr}$. In our simulation, the used factor is assumed to be 55 . This leads to an initial population of $\sim 385$ times the current belt mass for the entire Main Belt, which is consistent with the results obtained by Petit et al. (1999) and Petit et al. (2001) for the early asteroid belt.

Following Campo Bagatin et al. (1994a) and Campo Bagatin (1998), a collisional system with a low-mass cutoff leads to waves in the size distribution of the bodies. In order to avoid this effect, we do not evolve in time the 60 first size bins, whose central values range from $10^{-10}$ to $10^{-4} \mathrm{~km}$. For NEA population and each ring of the asteroid belt, this part of the population is only used as a tail of projectiles for calculating impact rates with larger bodies and its size distribution is determined each timestep by extrapolating the slope of the distribution of the ten next size bins.

In each timestep, a characteristic orbit is generated at random for each of the three rings of the asteroid Main Belt and NEA population for all the sizes. For the asteroid belt rings, we assign eccentricities $e$ between 0 and 0.3 , inclinations $i$ between 0 and $20^{\circ}$ and semimajor axes $a$ in such a way that $2 \leq$ $a \leq 2.5$ for the Inner Ring, $2.5 \leq a \leq 2.823$ for the Middle Ring and $2.823 \leq a \leq 3.27$ for the Outer Ring. In each case, the combination of $(a, i)$ values must be below the location of the $v_{6}$ resonance, while the combination of $(a, e)$ values must fall outside of the gaps associated with the 3:1 and 5:2 resonances, where the boundaries of such regions were already discussed in Sect. 4.1. For the NEA population, we use orbital parameters $0 \leq$ $a \leq 3.4,0 \leq e \leq 0.7$ and $0 \leq i \leq 40^{\circ}$ which are combined in such a way that the perihelion distance $q$ and the aphelion distance $Q$ are always smaller than 1.3 AU and larger than $0.983 \mathrm{AU}$, respectively, according to the definition of NEAs. Finally, given the longitude of ascending node $\Omega$, the argument of pericentre $\omega$ and the mean anomaly $M$ between 0 and $360^{\circ}$, an orbit can be assigned and from this, a position-velocity pair can be derived for all bodies of each population. In Sect. 5.1, we will discuss some aspects related to this treatment.

Once a typical orbit has been computed for each of the four populations of our model, the next step is to carry out the collisional treatment (including the analysis of the reaccumulation process) from the algorithm outlined in Sect. 2. In order to determine the final fate of the fragments escaping from the gravitational field of the system, it is necessary to calculate which are their orbital elements once they are ejected with a relative velocity with respect to the parent body. Immediately before the collision, the barycentric position and velocity of the fragments are assumed to be those associated with their parent body. After the collision, the relative velocity of the fragments with respect to the parent body (Eq. (15)) is assumed to be equally partitioned between the three components. Once the barycentric position and velocity of the fragments after the collision have been obtained, it is possible to calculate their orbital elements and the final fate of them. For this, we use the following criterion:

1. The fragments are placed in the NEA population, if any of the following conditions is fulfilled:

- the aphelion distance $Q \geq 0.983 \mathrm{AU}$ and the perihelion distance $q \leq 1.3 \mathrm{AU}$,

- the semimajor axis $a<2 \mathrm{AU}$,

- the combination of $(a, i)$ values falls above the location of the $v_{6}$ secular resonance, 
- the combination of $(a, e)$ values falls into the gap associated with the 3:1 mean motion resonance,

- the combination of $(a, e)$ values falls into the gap associated with the 5:2 mean motion resonance.

2. The fragments are placed in the Inner Ring of the Main Belt if

$-2 \leq a \leq 2.5 \mathrm{AU}$ and the combinations of $(a, e)$ values and $(a, i)$ values fall outside of the gap associated with the $3: 1$ resonance and below the location of the $v_{6}$ resonance, respectively.

3. The fragments are placed in the Middle Ring of the Main Belt if

$-2.5 \leq a \leq 2.823 \mathrm{AU}$ and the combinations of $(a, e)$ values and $(a, i)$ values fall outside of the gaps associated with the 3:1 and 5:2 resonances and below the location of the $v_{6}$ resonance, respectively.

4. The fragments are placed in the Outer Ring of the Main Belt if

$-2.823 \leq a \leq 3.27$ AU and the combinations of $(a, e)$ values and $(a, i)$ values fall outside of the gap associated with the 5:2 resonance and below the location of the $v_{6}$ resonance, respectively.

5. Finally, the fragments are assumed to be ejected from the Solar System on hyperbolic or parabolic orbits, no longer participating in the collisional evolution, if the eccentricity $e \geq 1$ or $a>3.27 \mathrm{AU}$.

Once the collisional treatment has been developed and all the collisional information has been kept, the removal rate of bodies due to the action of the Yarkovsky effect and orbital resonances must be included in our analysis. According to the dynamical results derived by Bottke et al. (2002) with regard to the different NEA source regions (Sect. 3.1), our algorithm assumes that the objects removed from the Inner, Middle and Outer Rings of the asteroid Main Belt are delivered to the NEA population. On the other hand, in order to take into account the mean time spent in the NEA region by bodies coming from the different source regions, it is necessary to include a rate of dynamical removal of objects from the NEA population. In a steady state, the rate of injection of bodies from a given source into the NEA population matches the rate of dynamical elimination of those bodies from such population. Thus, for any source

$\tau=\frac{N_{\mathrm{NEA}}}{T_{\mathrm{NEA}}}$

(Bottke et al. 2002), where $\tau$ gives the number of objects injected into the NEA region per unit time, $N_{\mathrm{NEA}}$ represents the steadystate number of NEAs and $T_{\mathrm{NEA}}$ is the median time spent in that population. These values are shown in Table 2. Following O'Brien \& Greenberg (2005), the mean dynamical lifetime of all bodies in the NEA population coming from the different source regions studied by Bottke et al. (2002) is given by

$\left\langle T_{\mathrm{NEA}}\right\rangle=\frac{\sum N_{\mathrm{NEA}}}{\sum \tau}$

where the summation extends to all the sources shown in Table 2, except the JFCs. Using the values given there, a $\left\langle T_{\mathrm{NEA}}\right\rangle$ of $1.14 \mathrm{Myr}$ is obtained. But, the numerical simulations performed by Migliorini et al. (1998) suggest that the multikilometer NEAs may have the Mars-crosser population as primary source. Thus, our model treats the dynamical removal of objects from the NEA population using a dynamical lifetime $T_{\mathrm{NEA}}^{\mathrm{IMC}}=3.75 \mathrm{Myr}$ associated with the Mars-crosser population for bodies with an $H$ magnitude smaller than 12 (namely, diameters larger than $\sim 15 \mathrm{~km}$ ) while $\left\langle T_{\mathrm{NEA}}\right\rangle$ is used for bodies with larger $H$ magnitudes.

To study the evolution in time of the populations, the timestep $\Delta t$ is calculated in such a way that the change of the number of objects in any size bin is always smaller than a given amount, which is generally chosen as $1 \%$ of the original number of bodies.

\section{Results}

In order to test the proposed model, here we compare our results to the most important observational constraints on the collisional and dynamical history of the asteroid Main Belt and NEA population. Thus, in Sect. 5.1, we compare our estimates of the Main Belt and NEA size distributions to observational data. Then, in Sect. 5.2, we discuss the relation between our estimate of the mean collisional lifetimes of bodies and the meteorite cosmicray exposure ages. In Sect. 5.3, we analyze our results in regard to the collisional history of Asteroid (4) Vesta. Then, we compare in Sect. 5.4 the results of our simulations with the number of asteroid families observed in the Main Belt. In Sect. 5.5, we analyze how the collisional process might contribute to the mixing of primordial material in the asteroid Main Belt. Finally, in Sect. 5.6, we study the provenance of the NEA objects.

\subsection{Main Belt and NEA size distributions}

The population of Main Belt asteroids is assumed to be reasonably complete to $\sim 30 \mathrm{~km}$ in diameter. Some years ago, several observational studies such as Spacewatch (Jedicke \& Metcalfe 1998), the Sloan Digital Sky Survey (SDSS) (Ivezić et al. 2001) and the Subaru Sub-km Main Belt Asteroid Survey (SMBAS) (Yoshida et al. 2003) were developed, which have allowed us to extend the Main Belt size distribution estimate down to a diameter of about $500 \mathrm{~m}$. As the reader can see in Fig. 7a, the estimated values of the asteroid Main Belt size distribution obtained from our simulations are in agreement with the observational data.

Figure $7 \mathrm{~b}$ shows our estimate of the NEA size distribution, which is described in terms of the absolute magnitude $H$. Following Bowell et al. (1989), it is possible to derive the diameter of a body with a given $H$-magnitude from the expression

$D=\frac{1347}{\sqrt{p_{\mathrm{v}}}} 10^{-H / 5}$

where $p_{\mathrm{v}}$ is the visual geometric albedo which is assumed to be 0.11 . The population of NEAs is believed to be observationally complete up to about $H=15$, which corresponds to a diameter of $\sim 4 \mathrm{~km}$. Several observational surveys have been developed in order to extend the NEA $H$-magnitude distribution up to larger $H$ values. In fact, Rabinowitz et al. (2000) used data obtained from Spacewatch and JPL's Near Earth Asteroid Tracking (NEAT) program for deriving an estimate of the NEA population down to a $H$ magnitude of $\sim 30$, while Stuart (2001) and Harris (2002) used the data from the LINEAR Survey in order to extend the NEA $H$-magnitude distribution down to $H$ magnitudes of 22.5 and 25.5, respectively. From Fig. 7b, it is possible to see that the NEA population resulting from our simulation fits to the observed data. One important result derived from our analysis is that the NEA $H$-magnitude distribution is determined primarily by the dynamical removal of asteroids from the Main Belt due to the action of the Yarkovsky effect and orbital resonances while the collisional processes do not play an important role. 


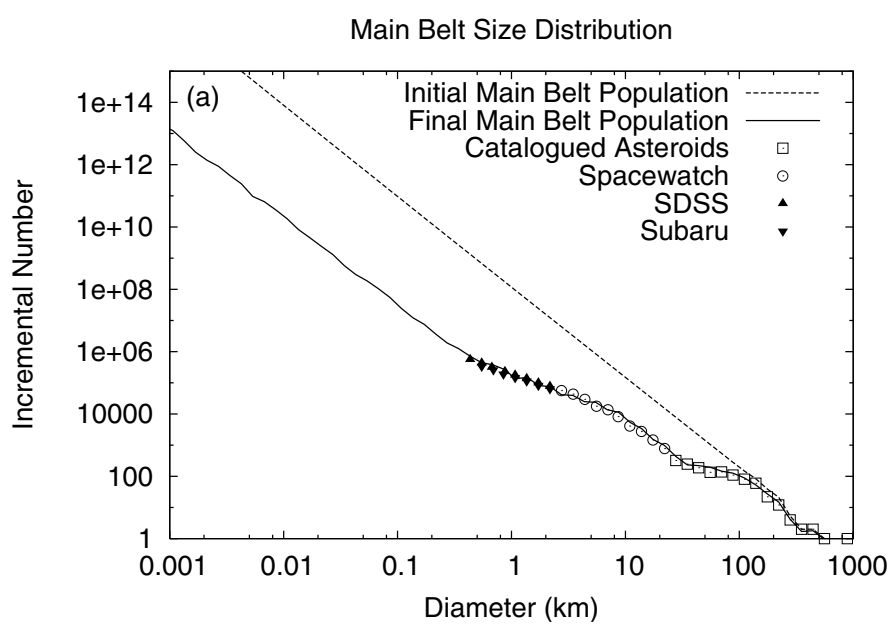

NEA H-Magnitude Distribution

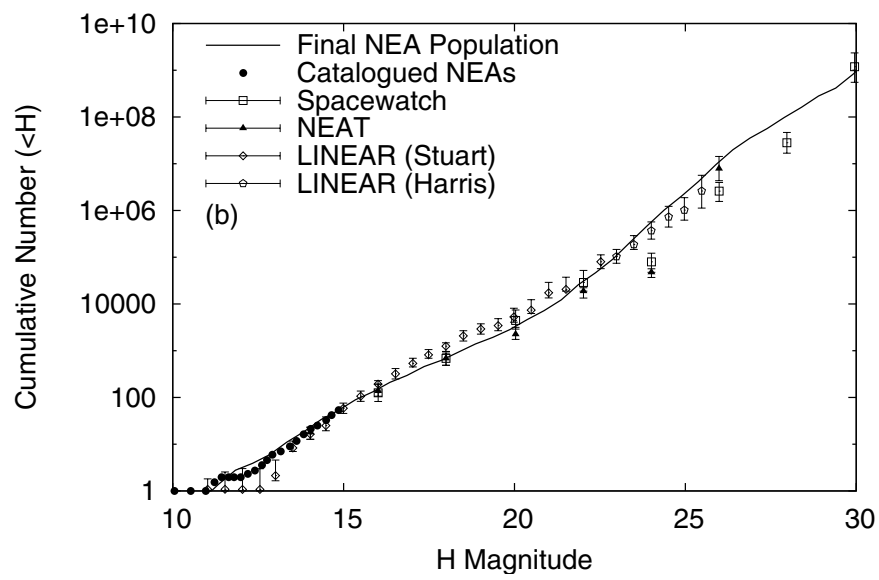

Fig. 7. Our estimates of the asteroid Main Belt size distribution a) and NEA H-magnitude distribution b). Observed data are given for comparison.

As we have already said in Sect. 4.5 and following O'Brien $\&$ Greenberg (2005), we include a brief period of primordial evolution at the beginning the simulation in order to reproduce the results of Petit et al. (1999) and Petit et al. (2001) concerning the mass loss from a massive early asteroid belt. In this phase, the initial populations associated with each of the three rings of the asteroid belt are multiplied by a factor of 55 and their evolution followed for 5 Myr. During this period, the intense collisional activity removes $\sim 74 \%$ of the initial mass of the Main Belt, leading to a residual initial population for the entire asteroid belt of $\sim 100$ times its current value. Then, the residual initial populations associated with each of the three rings of the Main Belt are reduced by that factor of 55 , which simulates the removal of about $98 \%$ of the masses of everyone of them. Finally, the evolution of a Main Belt initial population of $\sim 1.8$ times its current value is analyzed for the rest of the 4.5 Gyr. During this time, the collisional and dynamical mechanisms remove $\sim 25 \%$ and $\sim 17 \%$ of the initial mass of the Main Belt, respectively, leading to a final population for the entire asteroid belt of approximately its current value. On the other hand, our simulations suggest that the asteroid Main Belt population acquire a smooth wave structure similar to that observed in the current asteroid belt during the first $5 \mathrm{Myr}$ of evolution (see Fig. 8). These results are consistent with those obtained by Bottke et al. (2005a), who indicate that the Main Belt size distribution is predominately a fossil.

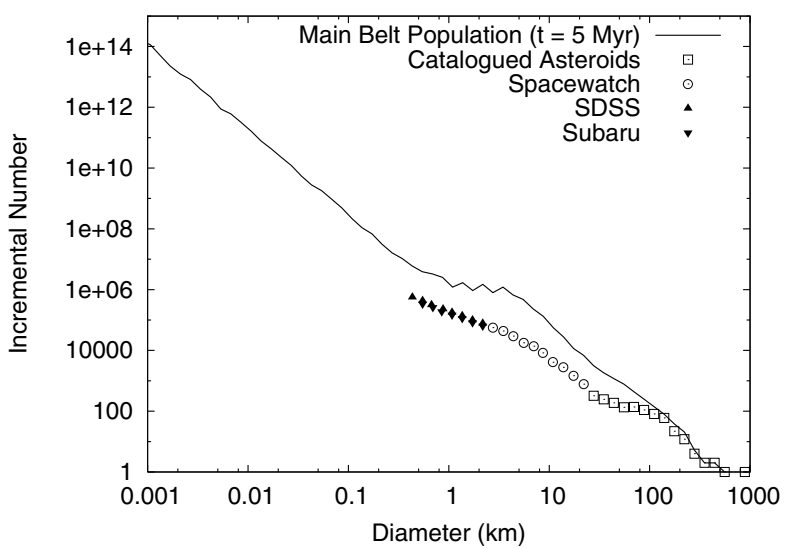

Fig. 8. Our estimate of the asteroid Main Belt Size Distribution after 5 Myr. A smooth wave structure is formed during the first 5 Myr of evolution.
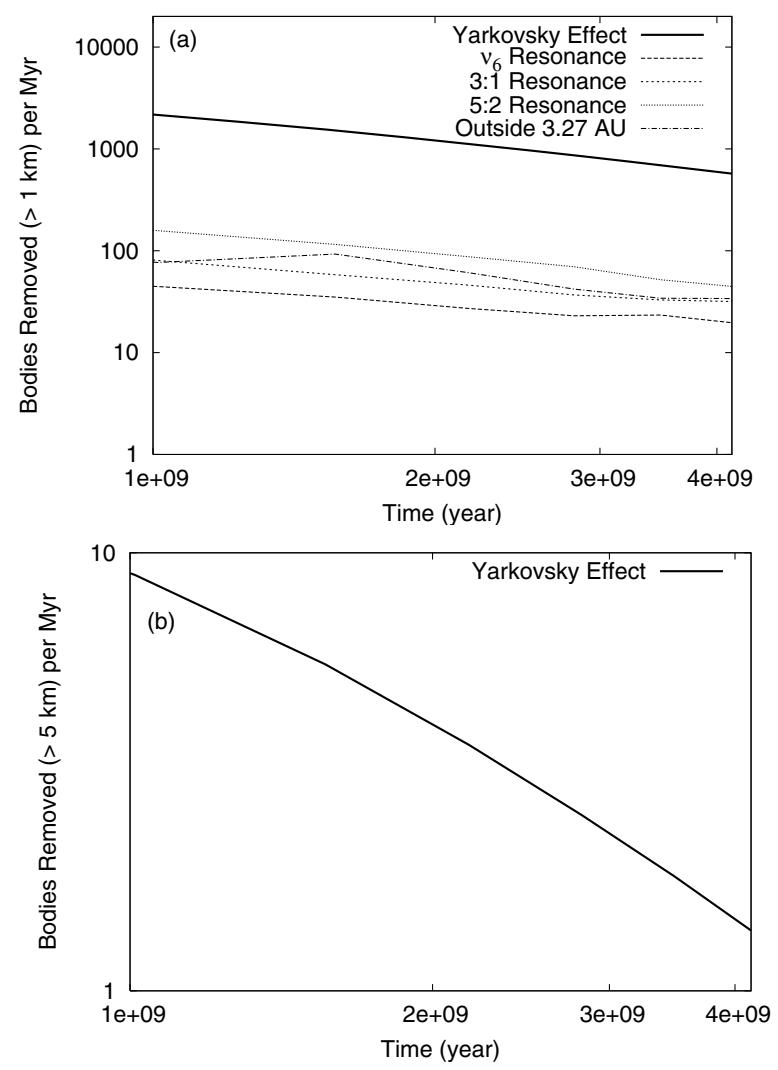

Fig. 9. a) Number of bodies of diameter $D \geq 1 \mathrm{~km}$ removed per unit time from the entire Main Belt over the history of the Solar System. Here, we include the asteroid removal due to the Yarkovsky effect, collisional injection into the $v_{6}, 3: 1$ and 5:2 resonances and collisional ejection outside 3.27 AU. A mean removal rate of 1070 asteroids larger than $1 \mathrm{~km}$ per Myr from the entire Main Belt is obtained, which is in agreement with the results derived by Bottke et al. (2002). b) Number of bodies of diameter $D \geq 5 \mathrm{~km}$ removed per unit time from the Inner Ring of the Main Belt over the history of the Solar System. Here, we just include the asteroid removal due to the action of the Yarkovsky effect since the collisional injection rate into the powerful resonances and the collisional ejection of material outside 3.27 AU are negligible. A mean removal rate of 3 asteroids larger than $5 \mathrm{~km}$ per Myr from the Inner Ring is obtained over the last $3 \mathrm{Gyr}$, which is in agreement with the analysis developed by Morbidelli \& Nesvorný (1999).

Figure 9 shows the kilometer-scale asteroid removal rates from the entire Main Belt and the Inner Ring, taking into account 
the action of the Yarkovsky effect, the collisional injection of material into the $v_{6}, 3: 1$ and 5:2 resonances and the collisional ejection outside $3.27 \mathrm{AU}$. Our results indicate that 1070 asteroids larger than $1 \mathrm{~km}$ are removed per Myr from the entire Main Belt, which is consistent with the analysis developed by Bottke et al. (2002). Besides, our study determines the escape of 3 asteroids larger than $5 \mathrm{~km}$ per Myr from the Inner Ring of the asteroid belt over the last $3 \mathrm{Gyr}$, which is in agreement with the work performed by Morbidelli \& Nesvorný (1999). Figure 9 allows us to infer that the Yarkovsky effect is the most important process that removes material from the asteroid Main Belt, rather than collisional injection into the major resonances, which is consistent with the works of Morbidelli et al. (2002) and Morbidelli \& Vokrouhlický (2003). In fact, while 891 asteroids larger than $1 \mathrm{~km}$ are removed per Myr due to the action of the Yarkovsky effect, the collisional processes inject a total of about 25, 38 and 68 asteroids larger $1 \mathrm{~km}$ per Myr into the $v_{6}, 3: 1$ and 5:2 resonances, respectively. These removal rates have been obtained following the dynamical treatment proposed in Sect. 4.5. From this, in each timestep only one characteristic orbit is considered in each zone for all the sizes. To test this assumption we also performed some simulations where, in each timestep, different orbits were generated at random for each group of bodies (of a given diameter $D$ ) in each zone. While the CPU time was much longer, the results did not show relevant changes.

\subsection{Cosmic ray exposure ages of meteorites}

The cosmic-ray exposure (CRE) ages of meteorites represent the time interval that a body was exposed to cosmic rays in space as a meter-sized object or near the surface of a larger body. Thus, CRE ages allow us to determine the time in space between the meteoroid's liberation from its parent body and its arrival at the Earth. According to Marti \& Graf (1992) and Morbidelli \& Gladman (1998), CRE ages for the different types of ordinary chondrites, which represent the most common class of meteorites, range from a few million years to about $100 \mathrm{Myr}$ with a mean age of approximately 10-20 Myr. Figure 10 shows the mean collisional lifetimes obtained from our simulations. For meter-sized objects belonging to the Inner, Middle and Outer Rings of the asteroid Main Belt, we estimate mean collisional lifetimes of about 3.2, 4.3 and $6.8 \mathrm{Myr}$, respectively, which are within of factor 2-3 of the mean CRE ages of stony meteorites. Moreover, our results are consistent with those derived by O'Brien \& Greenberg (2005) who obtained a lifetime of about $8 \mathrm{Myr}$ for meter-sized objects.

\subsection{Collisional history of asteroid (4) Vesta}

Asteroid (4) Vesta, with a diameter of approximately $500 \mathrm{~km}$, orbits the Sun at a distance of about 2.362 AU. This object represents one of the most peculiar cases of the Solar System since it is the only known differentiated asteroid with an intact basaltic crust (Keil 2002). We find that $D \sim 500 \mathrm{~km}$ asteroids in the Inner Ring of the Main Belt have a mean collisional lifetime of 17.7 Gyr (Fig. 10), which allows us to infer that an object like Vesta has $\sim 75 \%$ probability of surviving over Solar System history without receiving a catastrophic impact, which is in agreement with the preservation of the intact basaltic crust of this asteroid.

On the other hand, Hubble Space Telescope (HST) observations of Vesta have revealed the existence of a singular crater with a diameter of about $450 \mathrm{~km}$ on its surface. According to

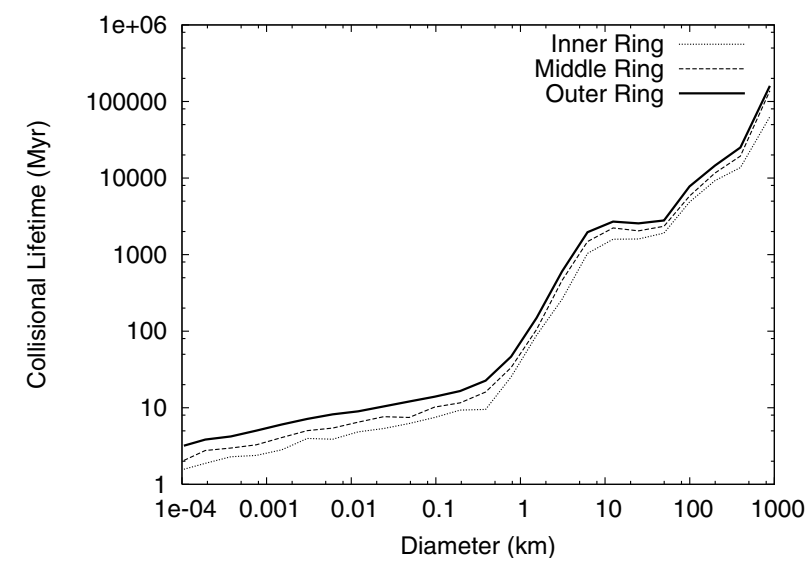

Fig. 10. Mean collisional lifetimes of bodies belonging to the Inner, Middle and Outer Rings of the asteroid Main Belt, estimated from our simulations. Meter-scale objects have mean collisional lifetimes between 3.2 and $6.8 \mathrm{Myr}$, which are consistent with CRE ages of meteorites.

studies developed by Thomas et al. (1997), the diameter of the impactor that created such crater was $D_{\mathrm{p}} \sim 35 \mathrm{~km}$. Bottke et al. (2005b) used the fact that Vesta does not have two such craters as a very specific constraint of the collisional history of this asteroid. Estimating the approximated number of projectiles $N$ with diameters $D_{\mathrm{p}}$ of $\sim 35 \mathrm{~km}$ and taking into account that the average interval between such impacts on Vesta can be calculated by

$\tau=\frac{4}{\sum P i_{\mathrm{c}}\left(D_{\text {Vesta }}+D_{\mathrm{p}}\right)^{2} N}$,

where the summation extends to all the populations of the model and $P i_{\mathrm{c}}$ is the correspondent intrinsic collision probability, it is possible to determine the mean number of collisions between $D_{\mathrm{p}} \sim 35 \mathrm{~km}$ objects and Vesta over the age of the Solar System. In fact, our simulations indicate that the mean number of bodies of $\sim 35 \mathrm{~km}$ in diameter impacting Vesta over $4.5 \mathrm{Gyr}$ is $\sim 0.5$. As Bottke et al. (2005b), this result suggests that the odds are slightly against asteroid (4) Vesta having an unique crater of size comparable to its total size, but very much against this particular object having two such singular craters.

\subsection{Asteroid families}

The existence of asteroid families represents a clear consequence of the collisional activity in the Main Belt. According to the works developed by Zappalà et al. (1995), there are a total of about 60 statistically significant asteroid clusters in proper element space, and it is possible to identify approximately 25 reliable families. Our simulations predict the formation of 8 asteroid families from parent bodies larger than $200 \mathrm{~km}$ in diameter, which is consistent with that discussed by Davis et al. (1985) who suggested the existence of 8 actual families formed from the breakup of parent bodies larger than $200 \mathrm{~km}$. Moreover, it is important to take into account that the 8 asteroid families generated in the model form after the brief of primordial evolution which is included to model the existence of a massive early asteroid belt.

On the other hand, the studies of asteroid families developed by Vokrouhlický et al. (2006) have shown that the typical dispersal velocity for $\sim 5 \mathrm{~km}$ fragments is of order of a few tens of meters per second. Following O'Brien \& Greenberg (2005) we assume a maximum value for the velocity of fragments of 


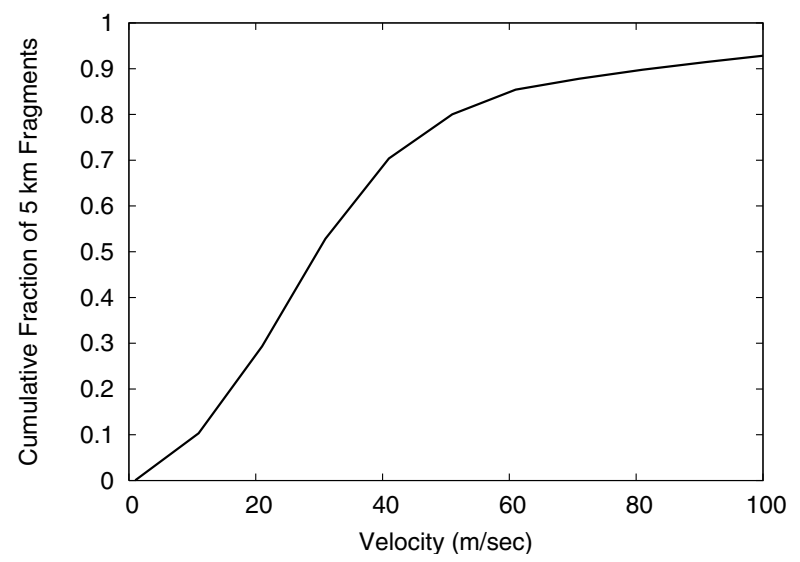

Fig. 11. Cumulative fraction of $\sim 5 \mathrm{~km}$ fragments as a function of velocity. Our study indicates that $\sim 90$ percent of the fragments of $5 \mathrm{~km}$ in diameter are ejected with velocities smaller than $80 \mathrm{~m} \mathrm{~s}^{-1}$, which is in agreement with Vokrouhlický et al. (2006).

$3000 \mathrm{~m} \mathrm{~s}^{-1}$, which is of order of the sound velocity in the material (see Sect. 2.4). While this value would seem to be too large, our studies indicate that $\sim 90 \%$ of the fragments of $5 \mathrm{~km}$ in diameter are ejected with velocities smaller than $80 \mathrm{~m} \mathrm{~s}^{-1}$ (see Fig. 11), which is in agreement with that discussed by Vokrouhlický et al. (2006).

\subsection{Mixing of taxonomic classes - Discussion}

Since some decades, the distribution of taxonomic classes in the Main Belt of asteroids has been thoroughly studied by many authors. For a long time, the work performed by Gradie \& Tedesco (1982) has been widely accepted as the major reference concerning the distribution and mixing of taxonomic classes. These authors studied a total of 656 objects with diameters larger than $50 \mathrm{~km}$ concluding that $S$-type asteroids represent the most abundant class in an inner zone between 2.1 and 2.5 AU, C-type asteroids dominate a central zone between 2.5 and 3.2 AU while $\mathrm{D} / \mathrm{P}$ types are the dominant classes in an outer zone after 3.2 AU. In addition, Gradie \& Tedesco (1982) showed the existence of some $\mathrm{C}$ and $\mathrm{D}$ asteroids in the inner zone and some $\mathrm{S}$ types in the outer zone. However, Mothé-Diniz et al. (2003) developed an analysis aimed at refining the heliocentric distribution of taxonomic types in the asteroid Main Belt. Using a total of 2026 objects with diameters larger than $13 \mathrm{~km}$, they found important differences with Gradie \& Tedesco (1982) and other previous works. In fact, Mothé-Diniz et al. (2003) concluded that S-type asteroids represent a significant fraction of the asteroid Main Belt population beyond $3 \mathrm{AU}$. Besides, they showed relevant discrepancies in the distribution of taxonomic classes considering different ranges of eccentricities and inclinations.

Knowing the existence of this distribution of taxonomies, the goal of this analysis is to determine if such distribution of classes is a characteristic feature of the Main Belt formation process or could have changed over the evolution of the Solar System. Figure 12 shows that, after 4.5 Gyr of evolution, more than 99 percent of the final mass of every ring is represented by primordial material. From this, we conclude that the distribution and mixing of taxonomic classes observed in the asteroid Main Belt can not be explained by the collisional exchange of mass and owing to that such distribution of taxonomies should be a primordial feature. In this study, the transport of material between the different regions of the Main Belt due to the
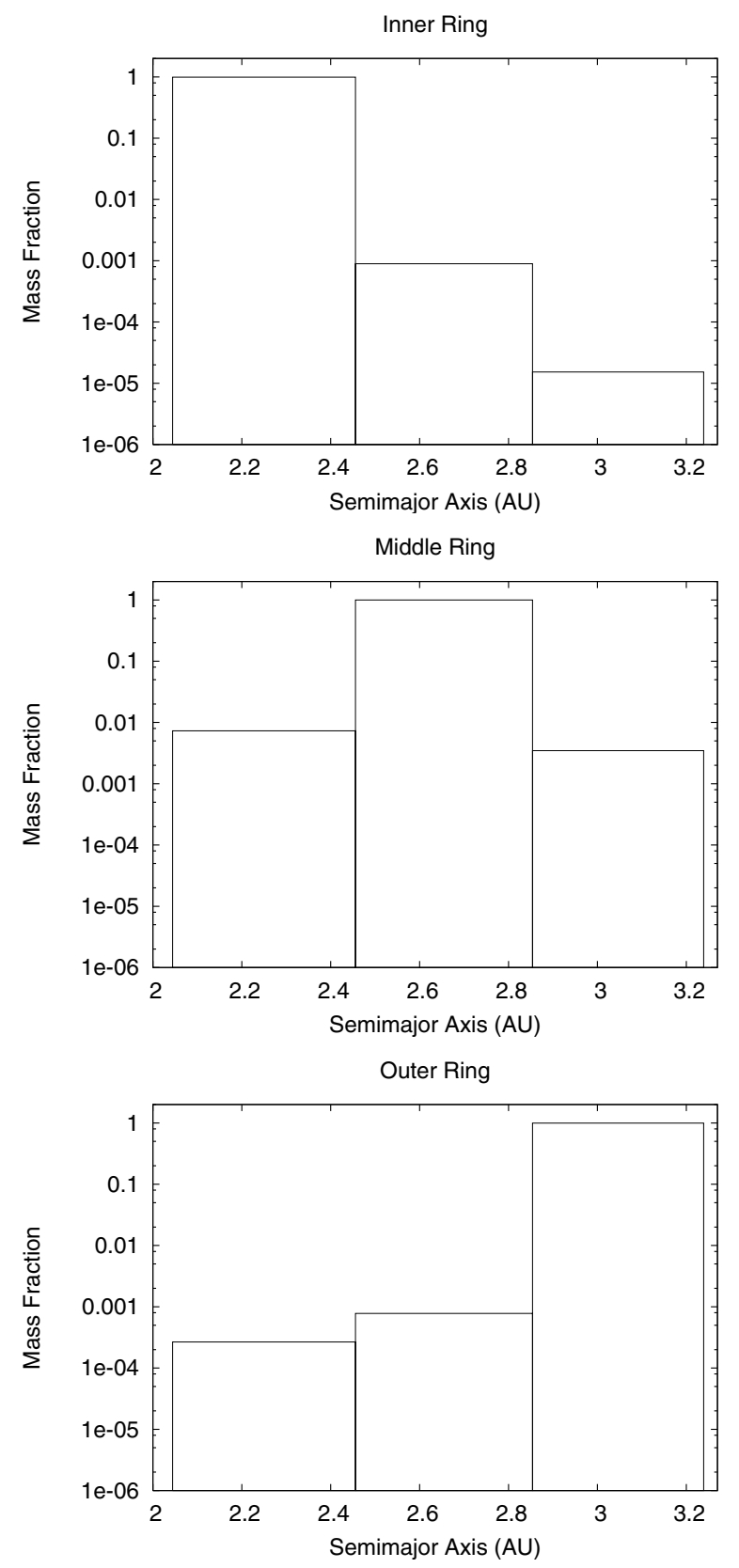

Fig. 12. Mass fraction of the Inner, Middle and Outer Rings distributed in the entire Main Belt due to the action of collisional processes after 4.5 Gyr. Our results indicate that each ring conserves more than 99 percent of its primordial mass which allows us to infer that the mixing of taxonomic classes observed in the asteroid belt can not be explained only by the collisional exchange of material.

action of the Yarkovsky effect has not been taken into account. In order to justify this assumption, we must analyze our model of the Yarkovsky effect together with the dynamical properties of the $v_{6}, 3: 1$ and 5:2 powerful resonances, which determine the boundaries of the rings of the Main Belt. Figure 4 shows that the semimajor-axis drift rate $\dot{a}$ of bodies $\geq 10^{-4} \mathrm{~km}$ is always smaller than $0.005 \mathrm{AU} \mathrm{Myr}^{-1}$ for any ring of the asteroid belt. In addition, as Table 1 indicates, the median lifetime of bodies initially in the $v_{6}$ and 3:1 resonances is $\sim 2 \mathrm{Myr}$ while, for the $5: 2$ resonance, the median lifetime is $\sim 0.5 \mathrm{Myr}$. Assuming that these powerful resonances have a characteristic width of some hundreds of an $\mathrm{AU}$, the time required to cross these regions is always larger than the median lifetimes. Thus, we consider that 
the Yarkovsky effect does not play an important role in mixing material between the different zones of the Main Belt.

\subsection{Provenance of the NEA objects}

Here, we study statistically what percentage of mass of the NEA population comes from the different regions of the Main Belt. In every timestep, we compute the injection rates from every ring of the asteroid belt into the NEA population and the rates of dynamical elimination of those bodies from such population. To calculate the influx rates, we take into account the action of the Yarkovsky effect and the collisional injection into the $v_{6}, 3: 1$ and 5:2 resonances. To compute the object removal rates from the NEA population, we need to determine a mean dynamical lifetime for bodies in this population. Following Bottke et al. (2002), we obtain a mean dynamical lifetime of 3.73 Myr in the NEA region for objects coming from the Inner and Middle Rings of the asteroid belt, averaging the values associated to the $v_{6} \mathrm{sec}-$ ular resonance, the intermediate-source Mars-crossing (IMC) population, and the 3:1 mean motion resonance. On the other hand, a mean dynamical lifetime of $0.14 \mathrm{Myr}$ is used for bodies coming from the Outer Ring of the Main Belt (Bottke et al. 2002, Table 2). Our analysis shows that $\sim 94 \%$ of the NEA population comes from the Inner and Middle Rings of the asteroid belt and $\sim 6 \%$ comes from the Outer Ring, which is in agreement with Bottke et al. (2002) who found that $\sim 85 \%$ of the NEA population comes from the inner and central Main Belt (namely, $a<$ $2.8 \mathrm{AU}), \sim 8 \%$ comes from the outer Main Belt and $\sim 6 \%$ comes from the Jupiter-family comet population.

\section{Conclusions}

We have presented a new multi-population code for collisional evolution that takes into account the main dynamical features present in the asteroid Main Belt and NEA region. The proposed collisional model is based on Petit \& Farinella's (1993) method that includes some corrections made by O'Brien \& Greenberg (2005). This algorithm allows us to describe the escape and reaccumulation processes of the fragments resulting from catastrophic fragmentation events and cratering impacts. The dynamical mechanisms taken into account in our code include mean motion and secular resonances, and the Yarkovsky effect, which represent a source of mass depletion in the asteroid belt and lead to a connection between the Main Belt and NEA populations.

While the previous works model the entire Main Belt, we study the collisional and dynamical evolution of the Main Belt and NEA populations, dividing the asteroid belt into three semimajor axis zones whose boundaries are given by the $v_{6}, 3: 1,5: 2$ and $2: 1$ resonances. This treatment allows us to calculate the direct collisional injection into these powerful resonances, to study the collisional exchange of mass between the different regions of the Main Belt and to analyze the provenance of the NEA objects. Our results are consistent with the predictions made by Morbidelli et al. (2002) and Morbidelli \& Vokrouhlický (2003), who proposed that the Yarkovsky effect is the most important process that removes material from the asteroid Main Belt, rather than collisional injection into the major resonances (Sect. 5.1). Besides, we conclude that the distribution and mixing of taxonomic classes observed in the asteroid belt (Mothé-Diniz et al. 2003) can not be explained by the collisional exchange of mass since more than 99 percent of the final mass of every of the three rings of our model of the Main Belt is represented by primordial material (Sect. 5.5). With regard to the provenance of the NEAs, our work shows that $\sim 94 \%$ of the NEA population comes from the Inner and Middle Rings of the asteroid belt and $\sim 6 \%$ comes from the Outer Ring (Sect. 5.6), which is in agreement with Bottke et al. (2002).

Our numerical algorithm have proved to satisfy the major observational constraints associated with the Main Belt and NEA populations, such as their size distributions, the collisional history of Vesta, the number of large asteroid families and the cosmic-ray exposure (CRE) ages of meteorites (Sect. 5). Besides, our model allows us to reproduce the dynamical results derived by Morbidelli \& Nesvorný (1999) and Bottke et al. (2002) with regard to the removal rate for multi-kilometer bodies from the Main Belt (Sect. 5.1).

Finally, this new multi-population code can be adapted in order to study the collisional and dynamical evolution of any small body population.

Acknowledgements. This work was partially financed by ANPCyT by grant PICT 03-11044. We also acknowledge to Ricardo Gil Hutton for valuable discussions during this work.

\section{References}

Benz, W., \& Asphaug, E. 1999, Icarus, 142, 5

Bottke, W. F., Morbidelli, A., Jedicke, R., et al. 2002, Icarus, 156, 399 Bottke, W. F., Durda, D. D., Nesvorný, D., et al. 2005a, Icarus, 175, 111 Bottke, W. F., Durda, D. D., Nesvorný, D., et al. 2005b, Icarus, 179, 63 Bowell, E., Hapke, B., Domingue, D., et al. 1989, in Asteroids II, ed. R. P. Binzel, T. Gehrels, \& M. S. Matthews (Tucson, USA: University of Arizona Press), 524

Brož, M., Vokrouhlický, D., Roig, F., et al. 2005, MNRAS, 359, 1437

Burns, J. A., Lamy, P. L., \& Soter, S. 1979, Icarus, 40, 1

Campo Bagatin, A. 1998, Ph.D. Thesis, University of Valencia, Spain

Campo Bagatin, A., Cellino, A., Davis, D. R., Farinella, P., \& Paolicchi, P. 1994a, Planet. Space Sci., 42, 1079

Campo Bagatin, A., Farinella, P., \& Petit, J. 1994b, Planet. Space Sci., 42, 1099

Davis, D. R., Chapman, C. R., Weidenschilling, S. J., \& Greenberg, R. 1985, Icarus, 62, 30

Davis, D. R., Weidenschilling, S. J., Farinella, P., Paolicchi, P., \& Binzel, R. P. 1989, in Asteroids II, ed. R. P. Binzel, T. Gehrels, \& M. S. Matthews (Tucson, USA: University of Arizona Press), 805

Dobrovolskis, A. R., \& Burns, J. A. 1984, Icarus, 57, 464

Dohnanyi, J. W. 1969, J. Geophys. Res., 74, 2531

Durda, D. D., Greenberg, R., \& Jedicke, R. 1998, Icarus, 135, 431

Farinella, P., Paolicchi, P., \& Zappalà, V. 1982, Icarus, 52, 409

Farinella, P., Vokrouhlický, D., \& Hartmann, W. K. 1998, Icarus, 132, 378

Fernández, J. A., Gallardo, T., \& Brunini, A. 2002, Icarus, 159, 358

Fujiwara, A., \& Tsukamoto, A. 1980, Icarus, 44, 142

Fujiwara, A., Kamimoto, G., \& Tsukamoto, A. 1977, Icarus, 31, 277

Gault, D. E., Shoemaker, E. M., \& Moore, H. J. 1963, NASA Tech. Note D-1767 Giblin, I. 1998, Planet. Space Sci., 46, 921

Giblin, I., Martelli, G., Smith, P. N., et al. 1994, Icarus, 110, 203

Gladman, B. J., Migliorini, F., Morbidelli, A., et al. 1997, Science, 277, 197

Gradie, J., \& Tedesco, E. 1982, Science, 216, 1405

Greenberg, R., Hartmann, W. K., Chapman, C. R., \& Wacker, J. F. 1978, Icarus, 35,1

Harris, A. W. 2002, BAAS, 34, 835

Hartmann, W. K. 1988, Lunar and Planetary Sci. XIX, 451

Holsapple, K. A. 1993, Annu. Rev. Earth Planet. Sci., 22, 333

Holsapple, K. A. 1994, Planet. Space Sci., 42, 1067

Housen, K. R. 1991, Icarus, 94, 180

Housen, K. R., \& Holsapple, K. A. 1990, Icarus, 84, 226

Housen, K. R., \& Holsapple, K. A. 1999, Icarus, 142, 21

Ivezić, Ž., Tabachnik, S., Rafikov, R., et al., and the SDSS Collaboration 2001, AJ, 122, 2749

Jedicke, R., \& Metcalfe, T. S. 1998, Icarus, 131, 245

Keil, K. 2002, in Asteroids III, ed. W. F. Bottke, A. Cellino, P. Paolicchi, \& R. P. Binzel (Tucson, USA: University of Arizona Press), 573

Knežević, Z., \& Milani, A. 2003, A\&A, 403, 1165

Love, S. G., \& Ahrens, T. J. 1996, Icarus, 124, 141

Marti, K., \& Graf, T. 1992, Annu. Rev. Earth Planet. Sci., 20, 221

Marzari, F., Scholl, H., \& Farinella, P. 1996, Icarus, 119, 192

Melosh, H. J. 1989 (New York: Oxford University Press)

Melosh, H. J., \& Ryan, E. V. 1997, Icarus, 129, 562

Michel, P., Migliorini, F., Morbidelli, A., \& Zappalà, V. 2000, Icarus, 145, 332 
Migliorini, F., Michel, P., Morbidelli, A., Nesvorný, D., \& Zappalà, V. 1998, Science, 281, 2022

Morbidelli, A., \& Gladman, B. 1998, Meteoritics and Planetary Science, 33, 999 Morbidelli, A., \& Henrard, J. 1991, Celest. Mechanics Dynamical Astron., 51, 131

Morbidelli, A., \& Nesvorný, D. 1999, Icarus, 139, 295

Morbidelli, A., \& Vokrouhlický, D. 2003, Icarus, 163, 120

Morbidelli, A., Bottke, W. F., Froeschlé, Ch., \& Michel, P. 2002, in Asteroids III, ed. W. F. Bottke, A. Cellino, P. Paolicchi, \& R. P. Binzel (Tucson, USA: University of Arizona Press), 409

Mothé-Diniz, T., Carvano, J. M., \& Lazzaro, D. 2003, Icarus, 162, 10

Murray, N., Holman, M., \& Potter, M. 1998, AJ, 116, 2583

Nakamura, A., \& Fujiwara, A. 1991, Icarus, 92, 132

Nakamura, A., Suguiyama, K., \& Fujiwara, A. 1992, Icarus, 100, 127

Nesvorný, D., \& Morbidelli, A. 1998, AJ, 116, 3029

Nesvorný, D., \& Morbidelli, A. 1999, Celest. Mechanics Dynamical Astron., 71, 243

Nesvorný, D., Ferraz-Mello, S., Holman, M., \& Morbidelli, A. 2002, in Asteroids III, ed. W. F. Bottke, A. Cellino, P. Paolicchi, \& R. P. Binzel (Tucson, USA: University of Arizona Press), 379

Nolan, M. C., Asphaug, E., Melosh, H. J., \& Greenberg, R. 1996, Icarus, 124, 359

O'Brien, D. P., \& Greenberg, R. 2005, Icarus, 178, 179

Penco, U., Dell'Oro, A., La Spina, A., et al. 2002, ACM Meeting, Berlin, Proc., ESA SP-500, Noordwijk, NL, 363

Peterson, C. 1976, Icarus, 29, 91
Petit, J., \& Farinella, P. 1993, Celest. Mechanics Dynamical Astron., 57, 1

Petit, J., Morbidelli, A., \& Valsecchi, G. B. 1999, Icarus, 141, 367

Petit, J., Morbidelli, A., \& Chambers, J. 2001, Icarus, 153, 338

Rabinowitz, D. L., Bowell, E., Shoemaker, E. M., \& Muinonen, K. 1994, Hazards Due to Comets and Asteroids, 285

Rabinowitz, D. L., Helin, E., Lawrence, K., \& Pravdo, S. 2000, Nature, 403, 165 Ryan, E. V. 1992, Ph.D. Thesis (Tucson, USA: University of Arizona)

Ryan, E. V., \& Melosh, H. J. 1998, Icarus, 133, 1

Stoeffler, D., Gault, D. E., Wedekind, J., \& Polkowski, G. 1975, J. Geophys. Res., 80,4062

Stuart, J. S. 2001, Science, 294, 1691

Tanaka, H., Inaba, S., \& Nakazawa, K. 1996, Icarus, 123, 450

Thomas, P. C., Binzel, R. P., Gaffey, M. J., et al. 1997, Science, 277, 1492

Vokrouhlický, D., Brož, M., Bottke, W. F., Nesvorný, D., \& Morbidelli, A. 2006, Icarus, 182, 118

Weidenschilling, S. J. 1977, Ap\&SS, 51, 152

Wetherill, G. W. 1979, Icarus, 37, 96

Williams, D. R., \& Wetherill, G. W. 1994, Icarus, 107, 117

Williams, J. G. 1969, Ph.D. Thesis, University of California, Los Angeles, USA

Wisdom, J. 1983, Icarus, 56, 51

Wisdom, J. 1985a, Icarus, 63, 272

Wisdom, J. 1985b, Nature, 315, 731

Yoshida, F., Nakamura, T., Watanabe, J., et al. 2003, PASJ, 55, 701

Zappalà, V., Bendjoya, P., Cellino, A., Farinella, P., \& Froeschlé, Ch. 1995, Icarus, 116, 291 